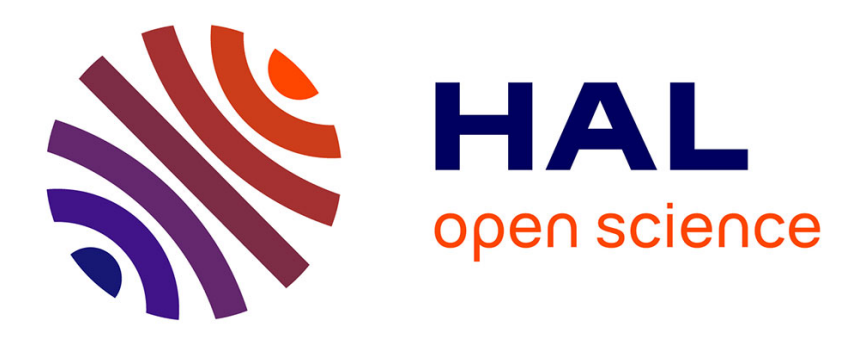

\title{
Characterizations of solutions for games with precedence constraints
}

\author{
Michel Grabisch, Peter Sudhölter
}

\section{To cite this version:}

Michel Grabisch, Peter Sudhölter. Characterizations of solutions for games with precedence constraints. International Journal of Game Theory, 2016, 10.1007/s00182-015-0465-y . hal-01297600

\section{HAL Id: hal-01297600 \\ https://hal.science/hal-01297600}

Submitted on 4 Apr 2016

HAL is a multi-disciplinary open access archive for the deposit and dissemination of scientific research documents, whether they are published or not. The documents may come from teaching and research institutions in France or abroad, or from public or private research centers.
L'archive ouverte pluridisciplinaire HAL, est destinée au dépôt et à la diffusion de documents scientifiques de niveau recherche, publiés ou non, émanant des établissements d'enseignement et de recherche français ou étrangers, des laboratoires publics ou privés. 


\title{
Characterizations of solutions for games with precedence constraints*
}

\author{
Michel GRABISCH ${ }^{\dagger} \quad$ Peter SUDHÖLTER ${ }^{\ddagger}$
}

December 12, 2014

\begin{abstract}
We generalize the characterizations of the positive core and the positive prekernel to TU games with precedence constraints and show that the positive core is characterized by non-emptiness (NE), boundedness (BOUND), covariance under strategic equivalence, closedness (CLOS), the reduced game property (RGP), the reconfirmation property (RCP) for suitably generalized Davis-Maschler reduced games, and the possibility of nondiscrimination. The bounded positive core, i.e., the union of all bounded faces of the positive core, is characterized similarly. Just RCP has to be replaced by a suitable weaker axiom, a weak version of CRGP (the converse RGP) has to be added, and CLOS can be deleted. For classical games the prenucleolus is the unique further solution that satisfies the axioms, but for games with precedence constraints it violates NE as well as the prekernel. The positive prekernel, however, is axiomatized by NE, anonymity, reasonableness, the weak RGP, CRGP, and weak unanimity for two-person games (WUTPG), and the bounded positive prekernel is axiomatized similarly by requiring WUTPG only for classical two-person games and adding BOUND.
\end{abstract}

Keywords: TU games, restricted cooperation, game with precedence constraints, positive core, bounded core, positive prekernel, prenucleolus

JEL Classification: C71

\section{Introduction}

Since the seminal paper of Faigle (1989) introducing the idea of restricted cooperation, that is, considering the possibility to have unfeasible or forbidden coalitions, many investigations have been done in order to study or adapt the main solution concepts of classical TU games, for various structures of the set of feasible coalitions.

Considering a finite set of players $N$ and a collection of feasible coalitions $\mathcal{F} \subseteq 2^{N}$, many structures borrowed from combinatorial optimization and partially ordered sets have been proposed for $\mathcal{F}$ : feasible coalitions induced by distributive lattices (Faigle and Kern 1992, Gilles, Owen, and van den Brink 1992,

\footnotetext{
${ }^{*}$ We are grateful to two anonymous referees of this journal for their remarks that helped to improve the writing of this paper. The first author thanks the Agence Nationale de la Recherche for financial support under contract ANR-13-BSHS10010. The second author acknowledges support from the Danish Council for Independent Research | Social Sciences under the FINQ project and from the Spanish Ministerio de Ciencia e Innovación under project ECO2012-33618, co-funded by the ERDF.

†Paris School of Economics, University of Paris I. 106-112, Bd. de l'Hôpital, 75013 Paris, France. Tel (+33)14407-8285, Fax (+33)14407-8301. Email: michel.grabisch@univ-paris1.fr

${ }^{\ddagger}$ Department of Business and Economics and COHERE, University of Southern Denmark. Campusvej 55, 5230 Odense M, Denmark. Email: psu@sam.sdu.dk
} 
Derks and Gilles 1995), convex geometries (Bilbao, Lebrón, and Jiménez 1998), union-stable systems (Algaba, Bilbao, Borm, and López 2000), antimatroids (Algaba, Bilbao, van den Brink, and JiménezLosada 2004), regular set systems (Lange and Grabisch 2009), to cite a few (see a survey in Grabisch 2012 ). The case of distributive lattices appears to be of special interest since it corresponds by Birkhoff's theorem to a situation which is often encountered in practice: the presence of a hierarchy, or any kind of partial order $\preceq$, on the set of players $N$. In this case, feasible coalitions are those satisfying the following rule: if player $i$ is present, then any of its subordinates $j \prec i$ must be present (this is called by Faigle and Kern "precedence constraints"). Moreover, the set of feasible coalitions is closed under union and intersection. For these reasons, we precisely focus in this paper on this type of structure ${ }^{1}$.

Considering that $\mathcal{F}$ is a distributive lattice induced by some partial order on $N$, the core of games on such structures has already deserved much attention, and many results valid for classical games have been extended to this case, in particular, the Shapley-Ichiishi theorem (Shapley 1971, Ichiishi 1981) characterizing convex TU games (Derks and Gilles 1995, Grabisch and Sudhölter 2014). The essential difference with classical games is that a nonempty core is always (unless $\mathcal{F}=2^{N}$ ) unbounded, however it is pointed. Extremal rays of the core have been found by Tomizawa (1983), and bounded facets have been studied by Grabisch (2011). The set of all bounded facets of the core, called the bounded core, has been studied and axiomatized by Grabisch and Sudhölter (2012). From the interpretation point of view, it is the set of core elements for which any player takes the maximum from its subordinates.

Other solution concepts like the nucleolus, the kernel, etc. have been much less studied in the context of restricted cooperation. It is the main purpose of this paper to fill this gap for games with precedence constraints. The excess of a coalition, i.e., the difference of its worth and its payoff, may be regarded as a measure of dissatisfaction, if it is positive, whereas a coalition is satisfied if its excess is not positive. However, the prenucleolus lexicographically minimizes the non-increasingly ordered vector of all excesses, and for an element of the prekernel, the maximum surplus of any pair of players is balanced, even if it is negative. The positive core (Orshan and Sudhölter 2010) and the positive prekernel (Sudhölter and Peleg 2000) are defined similarly - only a negative excess or negative surplus that cannot be regarded as dissatisfaction is not taken into account. It turns our that to consider these two solutions when precedence constraints are available has several advantages. The main advantages are:

- Both the positive core and the positive prekernel of a game with precedence constraints are nonempty, whereas the prenucleoulus as well as the prekernel do not exist in this framework.

- Both solutions, the positive core and the positive prekernel on the set of games with precedence constraints are characterized similarly, but in some sense simpler, by intuitive properties as for classical games.

\footnotetext{
${ }^{1}$ We mention here the dual view of distributive lattices, introduced by Gilles, Owen, and van den Brink (1992), and developed by, e.g., Derks and Gilles (1995) or van den Brink and Gilles (1996). There, hierarchies are interpreted as permission structures, in the sense that feasible coalitions are those who contain all superiors of its members, i.e., if $i$ is present and $i \prec j$, then $j$ must be present. Under this viewpoint, players must have their superiors present, who permit them to act. By contrast, under our viewpoint, feasible coalitions $S$ may be considered as teams to whom some task or project is assigned. The task can be achieved only if all subordinates are present in $S$, and $v(S)$ represents the benefit induced by the realization of the task by $S$. This point of view is discussed by Grabisch and Xie (2007) in detail.
} 
Indeed, we prove that the positive core can be axiomatized in a way which is very close to the classical case, up to suitably generalizing the axioms, namely by non-emptiness (NE), reasonableness (REAS), covariance (COV), the reduced game property (RGP), the reconfirmation property (RCP), nondiscrimination (ND), and closedness (CLOS), the latter permitting to eliminate the relative interior of the positive core as a candidate for the solution. The positive core being unbounded unless $\mathcal{F}=2^{N}$, we propose likewise the bounded positive core, which has the same intuitive interpretation as for the bounded core. We find that it can be axiomatized by NE, COV, RGP, RCP restricted to classical games, ND, boundedness (BOUND), and a variant of the converse reduced game property, called RCRGP. The bounded positive core contains a particular point, which can be considered as the prenucleolus of the game, since it coincides with the usual prenucleolus when $\mathcal{F}=2^{N}$. It lexicographically minimizes the excesses of all coalitions in $\mathcal{F}$ the complements of which are also in $\mathcal{F}$, and then lexicographically maximizes the remaining excesses so that they are non-positive (thereby keeping the idea that players should take the maximum of their subordinates, while guaranteeing minimal losses if any). Now, the positive prekernel of any game contains the positive core of the game. We show that it is characterized by NE, anonymity (AN), REAS, a weak RGP property, CRGP, and weak unanimity for 2-persons games (WUTPG).

The paper is organized as follows. Section 2 gives the basic material on partially ordered sets, the core, and the bounded core of games with precedence constraints. Section 3 introduces and characterizes the positive core. Section 4 is devoted to the bounded positive core and introduces the a possible substitute of the prenucleolus, for which a Kohlberg-like criterion is given. The positive prekernel and its axiomatization are addressed in Section 5. In Section 6 we show that, except ND and RCRGP, each of axioms employed in the various characterizations is logically independent of the remaining axioms. It is not known whether ND or ND and RCRGP are needed in the characterizations of the positive core or its bounded variant, respectively.

\section{Notation, Definitions, and Preliminaries}

A partially ordered set (poset) is a pair $(P, \preceq)$ such that $P$ is a nonempty finite set and $\preceq$ is a partial order on $P$, i.e., a reflexive, antisymmetric, and transitive binary relation on $P$. As usual, we write $x \preceq y$ for $(x, y) \in \preceq$ and use $x \prec y$ if $x \preceq y$ and $x \neq y$. If $x \prec y$ and there is no $z \in P$ such that $x \prec z \prec y$ then $y$ covers $x$, denoted by $x \prec y$. A chain in $(P, \preceq)$ is a sequence $\left(x_{0}, \ldots, x_{q}\right)$ such that $x_{0} \prec \cdots \prec x_{q}$ where $q$ is called the length of the chain. The height of a poset is the length of a longest chain. The height of $x \in P$, denoted by $h(x)$, is the maximal length of a chain from a minimal element to $x$.

Let $U,|U| \geqslant 3$, be a set, the universe of players. A coalition is a finite nonempty subset of $U$. Let $N$ be a coalition and $(N, \preceq)$ be a poset. Then $S \subseteq N$ is a downset of $(N, \preceq)$ if $i \in S$ and $j \preceq i$ implies $j \in S$. Denote by $\mathcal{O}(N, \preceq)$ the set of downsets of $(N, \preceq)$. Note that $(\mathcal{O}(N, \preceq), \subseteq)$ is a distributive lattice of height $^{2}|N|$. By Birkhoff's representation theorem the opposite statement is also true: If $\mathcal{F} \subseteq 2^{N}$ and $(\mathcal{F}, \subseteq)$ is a distributive lattice of height $|N|$, then there exists a poset $(N, \preceq)$ such that $\mathcal{F}=\mathcal{O}(N, \preceq)$.

\footnotetext{
${ }^{2} \mathrm{~A}$ poset $(P, \preceq)$ is a lattice if for any $x, y \in P$ their supremum, denoted $x \wedge y$, and infimum, denoted $x \vee y$, exist. A lattice is distributive if $\wedge$ and $\vee$ satisfy distributivity.
} 
A (cooperative TU) game with precedence constraints (Faigle and Kern 1992) is a triple $(N, \preceq, v)$ such that $N$ is a coalition, $(N, \preceq)$ is a poset, and $v: \mathcal{O}(N, \preceq) \rightarrow \mathbb{R}, v(\emptyset)=0$. Note that a classical TU game is a pair $(N, v)$ such that $v: 2^{N} \rightarrow \mathbb{R}, v(\emptyset)=0$. Hence, we may identify a game $(N, v)$ with $(N, \preceq, v)$ where $(N, \preceq)$ is the poset of height 0 . Let $\Gamma$ denote the set of TU games with precedence constraints.

Throughout this section let $(N, \preceq, v)$ be a game with precedence constraints and denote $\mathcal{F}=\mathcal{O}(N, \preceq)$. Let

$$
X^{*}(N, v)=\left\{x \in \mathbb{R}^{N} \mid x(N) \leqslant v(N)\right\} \text { and } X(N, v)=\left\{x \in \mathbb{R}^{N} \mid x(N)=v(N)\right\}
$$

denote the set of feasible and Pareto efficient feasible payoffs (preimputations), respectively. We use $x(S)=\sum_{i \in S} x_{i}(x(\emptyset)=0)$ for every $S \in 2^{N}$ and every $x \in \mathbb{R}^{N}$ as a convention. Additionally, $x_{S}$ denotes the restriction of $x$ to $S$, i.e. $x_{S}=\left(x_{i}\right)_{i \in S}$, and we write $x=\left(x_{S}, x_{N \backslash S}\right)$.

The core of $(N, \preceq, v)$, denoted by $C(N, \preceq, v)$, is defined by

$$
C(N, \preceq, v)=\left\{x \in \mathbb{R}^{N} \mid x(N)=v(N) \text { and } x(S) \geqslant v(S) \text { for all } S \in \mathcal{F}\right\} .
$$

By its definition, the core of $(N, \preceq, v)$ is a convex polyhedral set. It is well known (Derks and Gilles 1995) that it does not contain lines. More precisely,

$$
C(N, \preceq, v)=\operatorname{conv}(\operatorname{ext}(C(N, \preceq, v)))+C(N, \preceq, 0)
$$

where the game 0 assigns 0 to each element of $\mathcal{F}$, "conv" means "convex hull", "ext" means "set of extreme points", and "+" denotes "Minkowski sum". For any $S \subseteq N$, let ${ }^{N} \chi^{S}=\chi^{S} \in \mathbb{R}^{N}$ be the indicator function of $S$, i,e. $\chi_{i}^{S}=1$ for $i \in S$ and $\chi_{j}^{S}=0$ for $j \in N \backslash S$. If $(N, \preceq, v)$ is a classical game, i.e., if the height of $(N, \preceq)$ is 0 , then $C(N, \preceq, 0)=\{0\}$. Otherwise, i.e., if there exists a pair $(i, j) \in N \times N$ such that $i \prec j$, then (Derks and Gilles 1995)

$$
C(N, \preceq, 0)=\operatorname{cone}\left(\left\{\chi^{\{i\}}-\chi^{\{j\}} \mid i, j \in N, i \prec j\right\}\right),
$$

where "cone" denotes "convex cone generated by". For $i \prec j$, say $i=i_{0} \prec \cdots \prec \cdot i_{k}=j$,

$$
\chi^{\{i\}}-\chi^{\{j\}}=\sum_{\ell=0}^{k-1} \chi^{\left\{i_{\ell}\right\}}-\chi^{\left\{i_{\ell+1}\right\}}
$$

so that

$$
C(N, \preceq, 0)=\operatorname{cone}\left(\left\{\chi^{\{i\}}-\chi^{\{j\}} \mid i, j \in N, i \prec \cdot j\right\}\right)
$$

(also shown by Tomizawa (1983), see also Fujishige (2005, Th. 3.26)).

The bounded core of $(N, \preceq, v)$, denoted by $C^{b}(N, \preceq, v)$, is the set of all elements $x \in C(N, \preceq, v)$ that satisfy the following condition for any $i, j \in N$ with $i \prec \cdot j$ : There is no $\varepsilon>0$ such that $x+\varepsilon\left(\chi^{\{j\}}-\chi^{\{i\}}\right) \in$ $C(N, \preceq, v)$. Hence,

$$
C^{b}(N, \preceq, v)=\{x \in C(N, \preceq, v) \mid(\{x\}-C(N, \preceq, 0)) \cap C(N, \preceq, v)=\{x\}\} .
$$

Therefore, if $(N, \preceq, v)$ is a classical game, the bounded core coincides with the classical core. 
Remark 2.1 According to Rockafellar (1970, Section 18) a closed convex set is the disjoint union of the relative interiors of its faces. Hence, any element of $C^{b}(N, \preceq, v)$ is in the interior of some face of $C(N, \preceq, v)$. We conclude that $C^{b}(N, \preceq, v)$ is the disjoint union of the relative interiors of the bounded faces of $C(N, \preceq, v)$, i.e., $C^{b}(N, \preceq, v)$ is the union of all bounded faces of $C(N, \preceq, v)$. Thus, the bounded core is connected.

The bounded core may be non-convex and, hence, a proper subset of the convex hull of the extreme points of the core (called "convex part of the core") even if the poset is connected as Example 2.2 shows. We say that $i, j \in S \subseteq N$ are connected in $(S, \preceq)$ if there is a path in $S$ that connects $i$ and $j$, that is, if there exist $k \in \mathbb{N}$ and $i_{1}, \ldots, i_{k} \in N$ such that $i=i_{1}, j=i_{k}$, and, for each $\ell=1, \ldots, k-1$, either $i_{\ell} \prec i_{\ell+1}$ or $i_{\ell+1} \prec i_{\ell}$. Any $\emptyset \neq S \subseteq N$ may be partitioned into its connected components, and $S \subseteq N$ is connected if $S=\emptyset$ or $S$ consists of a single component.

Example 2.2 Let $N=\{1,2,3,4\}$ and $(N, \preceq, v)$ be defined by $i \prec 4$ for $i \in T=\{1,2,3\}$ and, for $S \in \mathcal{F}$, $v(S)=6$ if $|S \cap T|=2, v(N)=12$, and $v(S)=0$, otherwise. Then $(0,6,6,0),(6,0,6,0),(6,6,0,0) \in$ $C^{b}(N, \preceq, v)$, but the convex midpoint of these points $x=(4,4,4,0) \notin C^{b}(N, \preceq, v)$ because $(2,4,4,2) \in$ $C(N, \preceq, v)$, which can be obtained from $(4,4,4,0)$ by a transfer $(-2,0,0,2)$.

One can show that the vertices of $C^{b}(N, \preceq, v)$ are $(0,6,6,0),(6,0,6,0),(6,6,0,0)$ and $(3,3,3,3)$. Then, the bounded core is the union of the three segments between $(3,3,3,3)$ and each of the vertices $(0,6,6,0)$, $(6,0,6,0)$, and $(6,6,0,0)$. Indeed, any point in the segment between $(3,3,3,3)$ and $(0,6,6,0)$ has the form $(3 \alpha,-3 \alpha+6,-3 \alpha+6,3 \alpha)$, and any transfer $\varepsilon\left(\chi^{\{4\}}-\chi^{\{i\}}\right)$ for $i \prec 4$ would lead to a point outside the core. The same conclusion holds for the two other segments by symmetry. Now, consider the point $(3,3,5,1)$, which is the midpoint of $(0,6,6,0),(6,0,6,0)$ and $(3,3,3,3)$. Observe that a transfer $(0,0,-2,2)$ on this point would give $(3,3,3,3) \in C(N, \preceq, v)$, hence it does not belong to the bounded core. By symmetry again, any point in the relative interior of the convex hull of $(3,3,3,3)$ and any two of the vertices $(0,6,6,0),(6,0,6,0)$, and $(6,6,0,0)$ is outside the bounded core.

For any $S \in \mathcal{F}$ and $x \in \mathbb{R}^{N}$, let $e(S, x, v)=v(S)-x(S)$ be the excess at $x$.

For any $\alpha \in \mathbb{R}$ the $\alpha$-core of $(N, \preceq, v)$, denoted by $C_{\alpha}(N, \preceq, v)$, is the set

$$
C_{\alpha}(N, \preceq, v)=\{x \in X(N, v) \mid e(S, x, v) \leqslant \alpha \forall S \in \mathcal{F} \backslash\{\emptyset, N\}\} .
$$

We now generalize (2.2). Let $v^{\alpha}$ be defined by as $v^{\alpha}(S)=v(S)-\alpha$ for all $S \in \mathcal{F} \backslash\{\emptyset, N\}$. Then $C_{\alpha}(N, \preceq, v)=C\left(N, \preceq, v^{\alpha}\right)$ so that

$$
C_{\alpha}(N, \preceq, v)=\operatorname{conv}\left(\operatorname{ext}\left(C_{\alpha}(N, \preceq, v)\right)\right)+C(N, \preceq, 0) .
$$

The following lemma is a preparation for Proposition 2.4 that will be frequently used in the remainder (e.g., in Remark 3.2 and in the proof of Proposition 5.2).

Lemma 2.3 If $(N, \preceq)$ is connected (i.e., $N$ consists of a unique connected component) and $\alpha \in \mathbb{R}$, then $C_{\alpha}(N, \preceq, v) \neq \emptyset$. 
Proof: Let $w=v^{\alpha}$. By Lemma 3.2 of Grabisch and Sudhölter $(2012), \mathcal{C}(N, \preceq, w) \neq \emptyset$ and the proof is finished by $(2.5)$.

q.e.d.

Let $\left(N, \preceq^{*}\right)$ be the reverse partially ordered set (i.e., $i \preceq^{*} j$ iff $\left.j \preceq i\right)$. Note that $\mathcal{O}\left(N, \preceq^{*}\right)=\{S \subseteq$ $N \mid N \backslash S \in \mathcal{F}\}$. Hence, $(N, \preceq)$ is connected iff $\left(N, \preceq^{*}\right)$ is connected. The dual game of $(N, \preceq, v)$ is the game $\left(N, \preceq^{*}, v^{*}\right)$ defined by $v^{*}(S)=v(N)-v(N \backslash S)$ for all $S \in \mathcal{O}\left(N, \preceq^{*}\right)$. As for classical games, if $x \in X(N, v)$ and $S \in \mathcal{O}\left(N, \preceq^{*}\right)$, then

$$
e\left(S, x, v^{*}\right)=v^{*}(S)-x(S)=v(N)-v(N \backslash S)-x(N)+x(N \backslash S)=-e(N \backslash S, x, v) .
$$

Hence

$$
\mathcal{C}_{-\alpha}\left(N, \preceq^{*}, v^{*}\right)=\{x \in X(N, v) \mid e(S, x, v) \geqslant \alpha \forall S \in \mathcal{F} \backslash\{\emptyset, N\}\} \text { for all } \alpha \in \mathbb{R} .
$$

In order to generalize Lemma 2.3 and (2.5) to games that do not necessarily have a connected hierarchy, we define the intermediate game (see Owen (1977) for a similar construction) of $(N, \preceq, v)$ as follows. Denote by $\mathcal{R}^{(N, \preceq)}=\mathcal{R}$ the partition of $N$ whose elements are the connected components of $N$. In the present context $\mathcal{R}$ is considered as natural set of "a priori unions" and not a "coalition structure" à la Aumann and Drèze (1974), i.e., we do not consider "component feasible" payoffs. The intermediate game of $(N, \preceq, v)$ is the classical TU game $\left(\mathcal{R}, v_{\mathcal{R}}\right)$ defined by $v_{\mathcal{R}}(\mathcal{T})=v(\bigcup \mathcal{T})$ for all $\mathcal{T} \subseteq \mathcal{R}$. Let $\mathcal{O}_{0}(N, \preceq)=\mathcal{F}_{0}$ be the subset of all elements of $\mathcal{F}$ that are not unions of connected components. Hence, $\mathcal{F} \backslash \mathcal{F}_{0}=\{\bigcup \mathcal{T} \mid \mathcal{T} \subseteq \mathcal{R}\}$. Now, for any $y \in X\left(\mathcal{R}, v_{\mathcal{R}}\right)$ and $\alpha \in \mathbb{R}$ define the $\alpha$-core w.r.t. $y, C_{\alpha, y}(N, \preceq, v)$, by

$$
C_{\alpha, y}(N, \preceq, v)=\left\{x \in X(N, v) \mid x(T)=y_{T} \forall T \in \mathcal{R} \text { and } e(S, x, v) \leqslant \alpha \forall S \in \mathcal{F}_{0}\right\}
$$

By slightly abusing notation, for any $S \subseteq N, S \neq \emptyset$, the sub-poset of $(N, \preceq)$ on $S$, i.e., the intersection of $\preceq$ and $S \times S$, is denoted by $(S, \preceq)$. For simplicity, we denote the partition of connected components of $S, \mathcal{R}^{(S, \preceq)}$, by $\mathcal{R}(S)$ and the sublattice $\mathcal{O}(S, \preceq)=\{T \cap S \mid T \in \mathcal{F}\}$ by $\mathcal{F}(S)$.

Proposition 2.4 For any game $(N, \preceq, v)$ with precedence constraints, any preimputation $y$ of the intermediate game $\left(\mathcal{R}, v_{\mathcal{R}}\right)$, and any $\alpha \in \mathbb{R}$,

$$
C_{\alpha, y}(N, \preceq, v) \neq \emptyset \text { and } C_{\alpha, y}(N, \preceq, v)=\operatorname{conv}\left(\operatorname{ext}\left(C_{\alpha, y}(N, \preceq, v)\right)\right)+C(N, \preceq, 0)
$$

Proof: Let $\mathcal{F}_{0}=\mathcal{O}_{0}(N, \preceq)$ and $\mathcal{R}=\mathcal{R}^{(\mathcal{N}, \preceq)}$. To show non-emptiness we construct the components of some $x \in C_{\alpha, y}(N, \preceq, v)$ as follows. Let $\beta \geqslant(-\alpha)+\max \left\{v(S) \mid S \in \mathcal{F}_{0}\right\}-\min \{y(\mathcal{T}) \mid \mathcal{T} \subseteq \mathcal{R}\}$ and $\beta \geqslant 0$. For $Q \in \mathcal{R}$ define $\left(Q, \preceq, v^{Q}\right)$ by

$$
v^{Q}(S)= \begin{cases}0 & , \text { if } S=\emptyset \\ y_{Q} & , \text { if } S=Q \\ \beta & , \text { if } S \in \mathcal{F}(Q) \backslash\{\emptyset, Q\} .\end{cases}
$$

As $(Q, \preceq)$ is connected there exists $x_{Q} \in C\left(Q, \preceq, v^{Q}\right)$ by Lemma 2.3. Hence, $x(Q)=y_{Q}$ for all $Q \in \mathcal{R}$. Let $S \in \mathcal{F}_{0}$ and $\widehat{\mathcal{T}}=\{Q \in \mathcal{R} \mid Q \subseteq S\}$. Then there exists $\widehat{Q} \in \mathcal{R}$ such that $\emptyset \neq \widehat{Q} \cap S \neq S$. As $\beta \geqslant 0$,

$$
x(S)=x(S \cap \widehat{Q})+x(S \backslash \widehat{Q}) \geqslant \beta+y(\widehat{\mathcal{T}}) \geqslant-\alpha+v(S)+\max \{-y(\mathcal{T}) \mid \mathcal{T} \subseteq \mathcal{R}\}+y(\widehat{\mathcal{T}}) \geqslant-\alpha+v(S)
$$


Hence $v(S)-x(S) \leqslant \alpha$.

The observation that $z(Q)=0$ for any $z \in C(N, \preceq, 0)$ and any $Q \in \mathcal{R}$ implies the second statement of the proposition.

q.e.d.

Note that for any $y \in X\left(\mathcal{R}, v_{\mathcal{R}}\right)$,

$$
\mathcal{C}_{-\alpha, y}\left(N, \preceq^{*}, v^{*}\right)=\left\{x \in X(N, v) \mid x(T)=y_{T} \forall T \in \mathcal{R}, e(S, x, v) \geqslant \alpha \forall S \in \mathcal{F}_{0}\right\} \text { for all } \alpha \in \mathbb{R} .
$$

\section{The positive core}

This section is devoted to the positive core. In the first subsection we generalize its definition to games with precedence constraints, and in the second subsection we present a characterization by simple and intuitive properties.

\subsection{Definition of the positive core}

In order to expand the definition of the positive core (Orshan and Sudhölter 2010) to TU games with precedence constraints, we employ and recall Justman's (1977) notion of a "general nucleolus" (Schmeidler 1969).

Let $D$ be a finite nonempty set, $X$ be a set, let $h: X \rightarrow \mathbb{R}^{D}$, and denote $d:=|D|$. Define $\theta: X \rightarrow \mathbb{R}^{d}$ by

$$
\theta_{t}(x)=\max _{T \subseteq D,|T|=t} \min _{i \in T} h_{i}(x) \text { for all } x \in X \text { and all } t=1, \ldots, d,
$$

that is, for any $x \in X, \theta(x)$ is the vector, whose components are the numbers $h_{i}(x), i \in D$, arranged in non-increasing order. Let $\geq_{l e x}$ denote the lexicographical order of $\mathbb{R}^{d}$. The nucleolus of $h$ w.r.t. $X$, $\mathcal{N U C}(h, X)$, is defined by

$$
\mathcal{N U C}(h, X)=\left\{x \in X \mid \theta(y) \geq_{\text {lex }} \theta(x) \text { for all } y \in X\right\} .
$$

Let $(N, \preceq, v) \in \Gamma, \mathcal{F}=\mathcal{O}(N, \preceq), \mathcal{R}=\mathcal{R}^{(N, \preceq)}$, and recall that $\mathcal{F} \backslash \mathcal{F}_{0}=\{\bigcup \mathcal{T} \mid \mathcal{T} \subseteq \mathcal{R}\}$. Then the positive core of $(N, \preceq, v)$, denoted by $C_{+}(N, \preceq, v)$, is defined by

$$
C_{+}(N, \preceq, v)=\mathcal{N} \mathcal{U C}\left(\left(e(S, \cdot, v)_{+}\right)_{S \in \mathcal{F}}, X^{*}(N, v)\right)
$$

where $t_{+}=\max \{0, t\}$ for $t \in \mathbb{R}$. Let $x \in C_{+}(N, \preceq, v)$. Then $x \in X(N, v)$ (otherwise there are positive excesses and all of them could be diminished) and, $e(S, x, v) \leqslant 0$ for all $S \in \mathcal{F}_{0}$ (otherwise, if $e(S, x, v)>0$ for some $S \in \mathcal{F}_{0}$, then there exist $\ell \notin S \ni k, k \prec \cdot \ell$, and one could diminish this excess and the excesses of all $S^{\prime} \in \mathcal{F}_{0}, \ell \notin S^{\prime} \ni k$, by a transfer $t\left(\chi^{\{k\}}-\chi^{\{\ell\}}\right), t>0$, without changing the excesses of further coalitions). We conclude that

$$
C_{+}(N, \preceq, v)=\left\{x \in X(N, v) \mid(x(Q))_{Q \in \mathcal{R}} \in C_{+}\left(\mathcal{R}, v_{\mathcal{R}}\right) \text { and } e(S, x, v) \leqslant 0 \text { for all } S \in \mathcal{F}_{0}\right\} .
$$

It is well-known that for a classical TU game $(N, v)$ the prenucleolus, i.e., the set

$$
\mathcal{N U C}\left((e(S, \cdot, v))_{S \subseteq N}, X^{*}(N, v)\right),
$$


consists of a single element $\nu(N, v)$ (the prenucleolus point) and that

$$
C_{+}(N, v)=\left\{x \in X(N, v) \mid e(S, x, v)_{+}=e(S, \nu(N, v), v)_{+} \text {for all } S \subseteq N\right\}
$$

Now, let $\nu$ be the prenucleolus point of the intermediate game $\left(\mathcal{R}, v_{\mathcal{R}}\right)$ and define $(N, \preceq, w)$ by $w(\bigcup \mathcal{T})=$ $\nu(\mathcal{T})$ for all $\mathcal{T} \subseteq \mathcal{R}$ such that $e\left(\mathcal{T}, \nu, v_{\mathcal{R}}\right)>0$ and $w(S)=v(S)$ for all other $S \in \mathcal{F}$. By $(3.2)$, $C_{+}(N, \preceq, v)=C(N, \preceq, w)$ so that, by $(2.2)$,

$$
C_{+}(N, \preceq, v)=\operatorname{conv}(\operatorname{ext}(C(N, \preceq, w)))+C(N, \preceq, 0)
$$

and we may define the bounded positive core by

$$
C_{+}^{b}(N, \preceq, v)=C^{b}(N, \preceq, w) .
$$

We conclude this subsection by first remarking that Kohlberg's (1971) characterizations of the prenucleolus may suitably be modified for the positive core and secondly by briefly discussing various bargaining sets for games with precedence constraints.

Remark 3.1 The variant of the "Kohlberg (1971) criterion" for the positive core of classical games is still valid. For $(N, \preceq, v) \in \Gamma$ and $x \in \mathbb{R}^{N}$ and $\alpha \in \mathbb{R}$ let $\mathcal{D}(\alpha, x, v)=\{N\} \cup\{S \in \mathcal{F} \mid e(S, x, v) \geqslant \alpha\}$ where $\mathcal{F}=\mathcal{O}(N, \preceq, v)$. Recall that $\mathcal{D} \subseteq 2^{N}$ is balanced if there are balancing coefficients for $\mathcal{D}$, i.e., there are $\delta_{S}>0, S \in \mathcal{D}$ such that $\sum_{S \in \mathcal{D}} \delta_{S} \chi^{S}=\chi^{N}$. If $x \in X(N, v)$, then following statements are equivalent (Orshan and Sudhölter 2010, Theorem 3.4):

$$
\begin{aligned}
x & \in C_{+}(N, \preceq, v) \\
\alpha>0, y \in \mathbb{R}^{N}, y(N)=0, y(S) \geqslant 0 \forall S \in \mathcal{D}(\alpha, x, v) & \Longrightarrow y(S)=0 \forall S \in \mathcal{D}(\alpha, x, v) \\
\alpha>0 & \Longrightarrow \mathcal{D}(\alpha, x, v) \text { is balanced }
\end{aligned}
$$

We also note that the definitions of the classical bargaining set and its variants use the notion of "individual" objections. In a TU game $(N, \preceq, v)$ with precedence constraints a player $\ell \in N$ has no objection against any of her subordinates $k \prec \ell$ so that the Aumann-Davis-Maschler pre-bargaining set (Aumann and Maschler 1964, Davis and Maschler 1967), the reactive pre-bargaining set (Granot 1994), and the semireactive pre-bargaining set (Sudhölter and Potters 2001) consist of all preimputations $x$ so that $(x(Q))_{Q \in \mathcal{R}}$ belongs to the respective pre-bargaining set of the intermediate game $\left(\mathcal{R}, v_{\mathcal{R}}\right)$ and, moreover, $e(S, x, v) \leqslant 0$ for all $S \in \mathcal{F}_{0}$. For the Mas-Colell pre-bargaining (Mas-Colell 1989) of $(N, \preceq, v)$ we only have one inclusion: If $(y(Q))_{Q \in \mathcal{R}}$ belongs to the Mas-Colell pre-bargaining set of the intermediate game and if $e(S, x, v) \leqslant 0$ for all $S \in \mathcal{F}_{0}$, then $y$ belongs to the Mas-Colell pre-bargaining set of $(N, \preceq, v)$. However, the positive core is a subset of any of the mentioned pre-bargaining sets.

\subsection{Characterization of the positive core}

On classical games the prenucleolus and the positive core are the unique solutions that satisfy a collection of plausible properties. In this section we show that the positive core on the set of TU games with precedence constraints is characterized by these axioms. 
We first recall the mentioned axioms of a solution. Let $\mathcal{F}=\mathcal{O}(N, \preceq)$ wherever it occurs in this section. Let $(N, \preceq, v) \in \Gamma, \emptyset \neq S \subseteq N, x \in \mathbb{R}^{N}$, and let $\pi: N \rightarrow N$ be a bijection (a permutation of $N$ ). The reduced game of $(N, \preceq, v)$ w.r.t. $S$ and $x$ is the game $\left(S, \preceq, v_{S, x}\right)$ defined by

$$
v_{S, x}(T)= \begin{cases}0 & , \text { if } T=\emptyset, \\ v(N)-x(N \backslash S) & , \text { if } T=S, \\ \max \{v(P)-x(P \backslash T) \mid P \in \mathcal{F}, P \cap S=T\} & , \text { if } T \in \mathcal{F}(S) \backslash\{\emptyset, S\} .\end{cases}
$$

The permutation $\pi$ is a symmetry of $(N, \preceq, v)$ if $\{\pi(S) \mid S \in \mathcal{F}\}=\mathcal{F}$ and $v(\pi(S))=v(S)$ for all $S \in \mathcal{F}$. Let $S Y M(N, \preceq, v)$ denote the set of symmetries.

A solution is a mapping $\sigma$ that assigns a subset $\sigma(N, \preceq, v)$ of $X^{*}(N, v)$ to any $(N, \preceq, v) \in \Gamma$. Its restriction to a set $\Gamma^{\prime} \subseteq \Gamma$ is again denoted by $\sigma$. Moreover, a solution on $\Gamma^{\prime}$ is the restriction to $\Gamma^{\prime}$ of some solution. Hence, if we do not explicitly specify the domain of a solution, then the domain is $\Gamma$, the set of all games with precedence constraints. A solution $\sigma$ on $\Gamma^{\prime} \subseteq \Gamma$ satisfies

- non-emptiness (NE) if $\sigma(N, \preceq, v) \neq \emptyset$ for all $(N, \preceq, v) \in \Gamma^{\prime}$;

- Pareto optimality (PO) if $\sigma(N, \preceq, v) \subseteq X(N, v)$ for all $(N, \preceq, v) \in \Gamma^{\prime}$;

- Covariance under strategic equivalence (COV) if, for all $(N, \preceq, v),(N, \preceq, w) \in \Gamma^{\prime}, \alpha>0$, and $\beta \in$ $\mathbb{R}^{N}$, the following holds: If $w(S)=\alpha v(S)+\beta(S)$ for all $S \in \mathcal{F}$, then $\sigma(N, \preceq, w)=\alpha \sigma(N, \preceq, v)+\beta$;

- Anonymity (AN) if, for all $(N, \preceq, v) \in \Gamma^{\prime}$ and all bijective mappings $\pi: N \rightarrow N^{\prime}$ the following holds: If $\left(N^{\prime}, \preceq^{\prime}, \pi v\right) \in \Gamma^{\prime}$, where $\pi(i) \preceq^{\prime} \pi(j)$ iff $i \preceq j,(\pi v)(\pi(S))=v(S)$ for all $S \subseteq \mathcal{F}$, and $\pi(x)=y \in \mathbb{R}^{N^{\prime}}$ is defined by $y_{\pi(i)}=x_{i} \forall x \in \mathbb{R}^{N}, \forall i \in N$, then $\sigma\left(N^{\prime}, \preceq^{\prime}, \pi v\right)=\pi(\sigma(N, \preceq, v))$;

- reasonableness (REAS) if, for all $(N, \preceq, v) \in \Gamma^{\prime}$ and all $x \in \sigma(N, \preceq, v)$ the following property holds for each minimal element $k$ and each maximal element $\ell$ in $N$ :

$$
x_{k} \geqslant \min \{v(S \cup\{k\})-v(S) \mid S \in \mathcal{F}, k \notin S\} \text { and } x_{\ell} \leqslant \max \{v(S)-v(S \backslash\{\ell\}) \mid S \in \mathcal{F}, \ell \in S\}
$$

(note that for all $S \in \mathcal{F}$ and for any minimal $k \in N$ and maximal $\ell \in N$, also $S \cup\{k\}$ and $S \backslash\{\ell\}$ belong to $\mathcal{F}$ );

- closedness (CLOS) if $\sigma(N, \preceq, v)$ is a closed set for all $(N, \preceq, v) \in \Gamma^{\prime}$;

- reduced game property (RGP) if the following condition holds: If $(N, \preceq, v) \in \Gamma^{\prime}, \emptyset \neq S \subseteq N$, and $x \in \sigma(N, \preceq, v)$, then $\left(S, \preceq, v_{S, x}\right) \in \Gamma^{\prime}$ and $x_{S} \in \sigma\left(S, \preceq, v_{S, x}\right)$;

- reconfirmation property (RCP) if the following condition holds for every $(N, \preceq, v) \in \Gamma^{\prime}$, every $x \in \sigma(N, \preceq, v)$, and every $\emptyset \neq S \subseteq N$ : If $\left(S, \preceq, v_{S, x}\right) \in \Gamma^{\prime}$ and $y_{S} \in \sigma\left(S, \preceq, v_{S, x}\right)$, then $\left(y_{S}, x_{N \backslash S}\right) \in$ $\sigma(N, \preceq, v)$;

- the possibility of nondiscrimination (ND) if, for every $(N, \preceq, v) \in \Gamma^{\prime}$ with $\sigma(N, \preceq, v) \neq \emptyset$, there exists $x \in \sigma(N, \preceq, v)$ such that $\pi(x)=x$ for all $\pi \in S Y M(N, \preceq, v)$, i.e., $x$ is invariant under all symmetries. 
NE, PO, and CLOS are self-explanatory. COV requires that the solution has to be covariant under a change of unit (we call this scale COV), and covariant under an additional initial endowment to the players (called translation COV). AN means that the solution should be independent of the labeling of the players. REAS means that any player $k$ corresponding to a minimal element in the hierarchy should receive at least her minimal marginal contribution (in particular, at least $v(\{k\})$ ), while a player $\ell$ being a maximal element should not receive more than her maximal marginal contribution (in particular, not more than $v(N)-v(N \backslash\{\ell\}))$. RGP means that if $x$ is a solution element, then its restriction to a subset $S$ should be an element of the solution of the corresponding reduced game. Note that in all our characterization results we may replace the traditional RGP introduced by Peleg (1986) by the weaker property that only requires that the restriction to a subset belongs to the solution of the reduced game provided that this reduced game belongs to the class $\Gamma^{\prime}$ of games under consideration (because in our characterizations $\Gamma^{\prime}$ is always closed under taking reduced games). RCP means that from a solution element $x$ and a solution element $y$ of the reduced game on $S$, one can build another solution element by concatenating $y$ and $x_{N \backslash S}$. Lastly, ND means that it is possible to find a solution element invariant under all symmetries of the game, and thus not favoring any player.

The following important remark is interesting in its own right and used in the proof of Theorem 3.7, the main result of this section.

Remark 3.2 (1) Let $\Gamma^{\text {free }}$ be the set of classical TU games ${ }^{3}$. We recall (Orshan and Sudhölter 2010, Theorem 4.1) that, if $|U|=\infty$, there are precisely three solutions on $\Gamma^{\text {free }}$ that satisfy NE, REAS, $\mathrm{COV}, \mathrm{RGP}, \mathrm{RCP}$, and ND, namely the prenucleolus, the positive core $\left(C_{+}(\cdot)\right)$, or its relative interior, i.e., rint $C_{+}(\cdot)$. Here, for $(N, \preceq, v) \in \Gamma$ and with $\mathcal{R}=\mathcal{R}^{(N, \preceq)}$,

$$
\operatorname{rint} C_{+}(N, \preceq, v)=\left\{\begin{array}{l|c}
x \in C_{+}(N, \preceq v) & \begin{array}{c}
e(S, x, v)<0 \text { for } S \in \mathcal{F}_{0} \text { or } S=\bigcup \mathcal{T} \text { for some } \\
\mathcal{T} \subseteq \mathcal{R} \text { such that } e\left(\mathcal{T}, \nu\left(\mathcal{R}, v_{\mathcal{R}}\right), v_{\mathcal{R}}\right)<0
\end{array}
\end{array}\right\} .
$$

For classical TU games, the foregoing set is nonempty because it contains the prenucleolus point. Hence, by Proposition 2.4 it is also nonempty in the general case so that it indeed coincides with the relative interior of the positive core.

(2) Note that $C_{+}(\cdot)$ satisfies REAS. Indeed, let $(N, \preceq, v) \in \Gamma, x \in X(N, v)$, and $k, \ell \in N$ such that $k$ is minimal and $\ell$ is maximal. If $x_{k}<t:=\min _{S \in \mathcal{F}, k \notin S} v(S \cup\{k\})-v(S)$, then choose $\varepsilon>0$ such that $x_{k}+(|N|-1) \varepsilon<t$, define $y \in X(N, v)$ by $y_{k}=x_{k}+(|N|-1) \varepsilon$ and $y_{i}=x_{i}-\varepsilon$ for all $i \in N \backslash\{k\}$ and let $S \in \mathcal{F} \backslash\{\emptyset, N\}$. If $k \in S$, then $e(S, y, v) \leq e(S, x, v)-\varepsilon$ and if $k \notin S$, then $e(S, y, v)<e(S \cup\{k\}, x, v)$. As $e(\{k\}, x, v)>0, x \notin C_{+}(N, \preceq, v)$. Similarly it is shown that $x_{\ell}>\max _{S \in \mathcal{F}, \ell \in S} v(S)-v(S \backslash\{\ell\})$ implies that $x \notin C_{+}(N, \preceq, v)$.

(3) It is straightforward to verify that $C_{+}(\cdot)$ on any $\Gamma^{\prime} \subseteq \Gamma$ satisfies NE, PO, COV, AN, and CLOS.

We say that $\Gamma^{\prime} \subseteq \Gamma$ is closed under reduction if, for all $(N, \preceq, v) \in \Gamma^{\prime}, \emptyset \neq S \subseteq N$, and $x \in X(N, v)$, $\left(S, \preceq, v_{S, x}\right) \in \Gamma^{\prime}$.

\footnotetext{
${ }^{3}$ When cooperation is not restricted, i.e., all coalitions are feasible, we call a game unrestricted (free).
} 


\section{Lemma 3.3 Let $\Gamma^{\prime} \subseteq \Gamma$.}

(1) If $\Gamma^{\prime}$ is closed under reduction, then both $C_{+}(\cdot)$ and rint $C_{+}(\cdot)$ on $\Gamma^{\prime}$ satisfy $\mathrm{RGP}$.

(2) Both $C_{+}$and rint $C_{+}$on $\Gamma^{\prime}$ satisfy $R C P$.

Proof: For $C_{+}$, Remark 3.1 (3.5) implies (see Theorem 6.3.14 of Peleg and Sudhölter 2007) RGP, and in order to show RCP the relevant part of the proof of Theorem 6.3.14 of RCP may be literally copied. In the case of rint $C_{+}$we may proceed similarly as before to show RGP. The "Kohlberg criterion" has to be modified only inasmuch as $\alpha>0$ has to be replaced by $\alpha \geqslant 0$ in (3.5) and (3.6).

In order to show that $\operatorname{rint} C_{+}$satisfies $\operatorname{RCP}$, let $(N, \preceq, v) \in \Gamma, x \in \operatorname{rint} C_{+}(N, \preceq, v), \emptyset \neq S \subseteq N$, $y \in \operatorname{rint} C_{+}\left(S, \preceq, v_{S, x}\right), T \in \mathcal{F}=\mathcal{O}(N, \preceq)$, and put $z=\left(y, x_{N \backslash S}\right)$. We have to show that $e(T, z, v) \leqslant$ $e(T, x, v)_{+}$and that $e(T, x, v)<0$ implies $e(T, z, v)<0$. If $x(T \cap S)=y(T \cap S)$, then $e(T, x, v)=e(T, z, v)$. Hence, we may assume that $y(T \cap S) \neq x(T \cap S)$. Therefore $T \cap S \in \mathcal{F}(S) \backslash\{\emptyset, S\}$. Let $P \subseteq N \backslash S$ such that $(T \cap S) \cup P \in \mathcal{F}$ and $v_{S, x}(T \cap S)=v((T \cap S) \cup P)-x(P)$. By definition of the reduced game, $e((T \cap S) \cup P, z, v) \geqslant e(T, z, v)$. If $e((T \cap S) \cup P, z, v)>0$ or $e((T \cap S) \cup P, x, v)>0$, then by RCP of $C_{+}, e((T \cap S) \cup P, z, v)=e((T \cap S) \cup P, x, v)$ so that $x(T \cap S)=y(T \cap S)$ which was excluded. If $e((T \cap S) \cup P, z, v)=0$, then $e((T \cap S) \cup P, x, v)<0$ so that $e\left(S \cap T, x_{S}, v_{S, x}\right)<0=e\left(S \cap T, y, v_{S, x}\right)$ so that $y \notin \operatorname{rint} C_{+}\left(S, \preceq, v_{S, x}\right)$ by $\mathrm{RGP}$ of $\operatorname{rint} C_{+}$.

q.e.d.

Lemma 3.4 On any $\Gamma^{\prime} \subseteq \Gamma$ the positive core satisfies ND.

Proof: For any $(N, \preceq, v) \in \Gamma^{\prime}, x \in C_{+}(N, \preceq, v)$, and $\pi \in \operatorname{SYM}(N \preceq, v)$, by AN also $\pi(x) \in C_{+}(N, \preceq, v)$. By convexity of the positive core, $y=\sum_{\pi \in \operatorname{SYM}(N, \preceq, v)} \frac{\pi(x)}{|\operatorname{SYM}(N, \preceq, v)|} \in C_{+}(N, \preceq, v)$. Finally, $y$ is invariant under symmetries.

q.e.d.

Lemma 3.5 If $\sigma$ is a solution that satisfies $\operatorname{REAS}$ and $\operatorname{RGP}$, then $e(S, x, v) \leqslant 0$ for all $(N, \preceq, v) \in \Gamma, x \in$ $\sigma(N, \preceq, v)$, and all $S \in \mathcal{O}_{0}(N, \preceq)$.

Proof: Let $x \in \sigma(N, \preceq, v), T \in \mathcal{O}_{0}(N, \preceq)$, and assume, on the contrary, that $e(T, x, v)>0$. Then there exist $k \in T$ and $\ell \in N \backslash T$ such that $k \prec \ell$. Now, $v_{\{k, \ell\}, x}(\{k\}) \geqslant v(T)-x(T \backslash\{k\})$ and, by RGP, $x_{\{k, \ell\}} \in \sigma\left(\{k, \ell\}, \preceq, v_{\{k, \ell\}, x}\right)$ so that REAS is violated.

q.e.d.

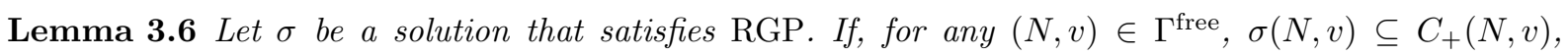
and if, for any $(N, \preceq, v) \in \Gamma$ and any $x \in \sigma(N, \preceq, v)$, e(T,x,v) $\leqslant 0$ for all $T \in \mathcal{O}_{0}(N, \preceq)$, then $\sigma$ is a subsolution of the positive core, i.e., $\sigma(N, \preceq, v) \subseteq C_{+}(N, \preceq, v)$ for $(N, \preceq, v) \in \Gamma$.

Proof: Let $S \subseteq N$ such that $|Q \cap S|=1$ for all $Q \in \mathcal{R}$. For any $i \in S$ denote $Q_{i}$ the connected component that contains $i$. Hence, the mapping $S \rightarrow \mathcal{R}, i \mapsto Q_{i}$ for all $i \in S$, is a bijection. As $\mathcal{F}(S)=2^{S}$, by RGP and our assumption, $x_{S} \in C_{+}\left(S, \preceq, v_{S, x}\right)$. Now, let $y \in \mathbb{R}^{\mathcal{R}}$ be defined by $y_{Q}=x(\bigcup Q)$ for all $Q \in \mathcal{R}$. Then, for any $T \subseteq S, e\left(T, x_{S}, v_{S, x}\right)_{+}=e\left(\left\{Q_{i} \mid i \in T\right\}, y, v_{\mathcal{R}}\right)_{+}$. Indeed, $v_{S, x}(T)=v(T \cup P)-x(P)$ 
for some $P \subseteq N \backslash S$ such that $T \cup P \in \mathcal{F}$. If $e\left(T, x_{S}, v_{S, x}\right)>0$ then $T \cup P$ must be a union of connected components, hence $T \cup P=\bigcup_{i \in T} Q_{i}$ so that the foregoing equation is valid. Conversely, if $e\left(\left\{Q_{i} \mid i \in T\right\}, y, v_{\mathcal{R}}\right)>0$, then $e\left(\left\{Q_{i} \mid i \in T\right\}, y, v_{\mathcal{R}}\right)=v\left(\bigcup_{i \in T} Q_{i}\right)-x\left(\bigcup_{i \in T} Q_{i}\right) \leqslant e\left(T, x_{S}, v_{S, x}\right)$.

By Remark 3.1 (3.5), $y \in C_{+}\left(\mathcal{R}, v_{\mathcal{R}}\right)$. The proof is finished by (3.1).

q.e.d.

Theorem 3.7 Assume that $|U|=\infty$. There is a unique solution that satisfies NE, REAS, COV, CLOS, $\mathrm{RGP}, \mathrm{RCP}$, and $\mathrm{ND}$, and it is the positive core.

Proof: The positive core satisfies the desired axioms by Remark 3.2, Lemma 3.3, and Lemma 3.4.

In order to show the remaining direction, let $\sigma$ be a solution that satisfies the desired axioms. On $\Gamma^{\text {free }}, \sigma$ must be one of the following three solutions (Orshan and Sudhölter 2010, Theorem 4.1): The prenucleolus, the positive core, or its relative interior. Hence, $\sigma$ is a subsolution of the positive core by Lemmas 3.5 and 3.6.

Step 1: We claim that, on $\Gamma^{\text {free }}, \sigma$ is the positive core. In order to show this statement it suffices to construct a single $\mathrm{TU}$ game $(M, u) \in \Gamma^{\text {free }}$ such that $\sigma(M, u) \backslash \operatorname{rint} C_{+}(M, u) \neq \emptyset$. For this purpose, choose any set $M^{\prime}$ of three players, say without loss of generality, $M^{\prime}=\{1,2,3\}$, and let $\preceq^{\prime}$ be defined by $1 \prec^{\prime} 2$. Moreover, let $\left(M^{\prime}, \preceq^{\prime}, u^{\prime}\right) \in \Gamma$ be defined by $u^{\prime}(M)=2$ and $u^{\prime}(S)=0$ for $S \in \mathcal{F}^{\prime} \backslash\left\{M^{\prime}\right\}$, where $\mathcal{F}^{\prime}=\mathcal{O}\left(M^{\prime}, \preceq^{\prime}\right)$. Let $P=\{1,3\}, Q=\{2,3\}$, and $R=\{1,2\}$. By NE, there exists $x \in \sigma\left(M^{\prime}, \preceq^{\prime}, u^{\prime}\right)$. As $x \in C_{+}\left(M^{\prime}, \preceq^{\prime}, u^{\prime}\right), x_{1}, x_{3} \geqslant 0, x_{3} \leqslant 2$, and $x\left(M^{\prime}\right)=2$. Note that all reduced games of $\left(M^{\prime}, \preceq^{\prime}, u^{\prime}\right)$ w.r.t. $P$ or $Q$ belong to $\Gamma^{\text {free }}$. By RGP, $\left(x_{2}, x_{3}\right) \in \sigma\left(Q, u_{Q, x}^{\prime}\right)$. As $u_{Q, x}^{\prime}(\{2\})=-x_{1}, u_{Q, x}^{\prime}(\{3\})=0$, $\left(1-x_{1}, 1\right)$ is the prenucleolus of $\left(Q, u_{Q, x}^{\prime}\right)$. As $\sigma\left(Q, u_{Q, x}^{\prime}\right)$ contains the prenucleolus, RCP implies that $y=\left(x_{1}, 1-x_{1}, 1\right) \in \sigma\left(M^{\prime}, \preceq^{\prime}, u^{\prime}\right)$. Now, if $x_{1}=0$, then by RGP $(0,1) \in \sigma\left(P, u_{P, y}^{\prime}\right)$. However, $(0,1) \in$ $C_{+}\left(P, u_{P, y}^{\prime}\right) \backslash \operatorname{rint} C_{+}\left(P, u_{P, y}^{\prime}\right)$. If $x_{1}>0$, then by RGP, $y_{R} \in \sigma\left(R, \preceq^{\prime}, u_{R, y}^{\prime}\right)$. As $u_{R, y}^{\prime}(\{1\})=0$ and $u_{R, y}^{\prime}(R)=1$, by (translation) $\operatorname{COV}\left(x_{1},-x_{1}\right) \in \sigma\left(R, \preceq^{\prime}, 0\right)$ and by (scale) $\operatorname{COV},(t,-t) \in \sigma\left(R, \preceq^{\prime}, 0\right)$ for all $t>0$ (choose $\alpha=\frac{t}{x_{1}}$ in the definition of scale COV). By CLOS, $(0,0) \in \sigma\left(R, \preceq^{\prime}, 0\right)$ so that by translation COV and RCP, $z=(0,1,1) \in \sigma\left(M^{\prime}, \preceq^{\prime}, u^{\prime}\right)$. Finally, RGP yields that $(0,1) \in \sigma\left(P, u_{P, z}^{\prime}\right)$ and $(0,1) \in C_{+}\left(P, u_{P, z}^{\prime}\right) \backslash \operatorname{rint} C_{+}\left(P, u_{P, z}^{\prime}\right)$.

Let $(N, \preceq, v) \in \Gamma$. It remains to show that

$$
C_{+}(N, \preceq, v) \subseteq \sigma(N, \preceq, v) .
$$

Let $\mathcal{F}=\mathcal{O}(N, \preceq, v)$. By Step 1 we may assume that $\mathcal{F} \neq 2^{N}$.

Step 2: We consider the case $|N|=2$ first and assume without loss of generality that $N=\{1,2\}$ and $1 \prec 2$. By COV we may assume that $v(S)=0$ for all $S \in \mathcal{F}$. With $\left(M^{\prime}, \preceq^{\prime}, u^{\prime}\right)$ defined in Step 1 , by NE there exists $y \in \sigma\left(M^{\prime}, \preceq^{\prime}, u^{\prime}\right)$. As in Step 1 we may assume that $y_{3}=1$. By RGP and RCP applied to $R$, we may assume that $y_{2} \geqslant 0$. Furthermore, by RGP and RCP applied to $P$, we may replace $\left(y_{1}, y_{3}\right)$ by $\left(y_{1}+y_{3}, 0\right)$ ( or by $\left(0, y_{1}+y_{3}\right)$ if necessary because $\left.\sigma\left(P, u_{P, y}^{\prime}\right)=C_{+}\left(P, u_{P, y}^{\prime}\right)\right)$. Thus, there exists $z, z^{\prime} \in \sigma\left(M^{\prime}, \preceq^{\prime}, u^{\prime}\right)$ with $z_{1}>0$ and $z_{1}^{\prime}=0$. By RGP and $\operatorname{COV},\left(z_{1},-z_{1}\right),(0,0) \in \sigma(N, \preceq, v)$ so that by scale $\operatorname{COV},(t,-t) \in \sigma(N, \preceq, v)$ for all $t \geqslant 0$, i.e., $\sigma(N, \preceq, v)=C_{+}(N, \preceq, v)$.

Now the proof of (3.3) can be finished by induction on $|N|$. For $|N|=1, C_{+}(N, \preceq, v)$ is a singleton so that 
(3.3) holds by NE and Lemmas 3.5 and 3.6. For $|N|=2$, Step 2 shows (3.3). Assume that (3.3) is proved whenever $|N| \leqslant k$ for some $t \in \mathbb{N}$ with $t>1$. If $|N|=t+1$, we may assume by Step 1 that $\mathcal{R}=\mathcal{R}^{(N, \preceq)}$ contains a non-singleton $Q$. Let $k \in Q$ be minimal. Let $A=\left\{x_{k} \mid x \in C_{+}(N, \preceq, v)\right\}$. Then $A=[\alpha, \infty[$ for some $\alpha \in \mathbb{R}$. Indeed, by REAS, $A$ has a lower bound and by closedness, there is a largest lower bound $\alpha$. Moreover, let $\ell \in Q$ with $k \prec \ell$. Replacing $x_{k}$ by $x_{k}+t$ and $x_{\ell}$ by $x_{\ell}-t$ for any $t \geqslant 0$ yields another element of $C_{+}(N, \preceq, v)$. Similarly, if $y \in \sigma(N, \preceq, v)$, then by RCP applied to $\{k, \ell\}$, we may replace $y_{k}$ by $y_{k}+t$ and $y_{\ell}$ by $y_{\ell}-t$ and receive another element of $\sigma(N, \preceq, v)$. Also, by applying RCP to the coalition $N^{\prime}=N \backslash\{k\}$, by the inductive hypothesis, $y^{\prime}=\left(y_{k}, z_{N^{\prime}}\right) \in \sigma(N, \preceq, v)$ for any $z_{N^{\prime}} \in C_{+}\left(N^{\prime}, \preceq, v_{N^{\prime}, y}\right)$. By RGP and RCP of $C_{+}, C_{+}\left(N^{\prime}, \preceq, v_{N^{\prime}, y}\right)=\left\{z_{N^{\prime}} \in \mathbb{R}^{N^{\prime}} \mid\left(y_{k}, z_{N^{\prime}}\right) \in C_{+}(N, \preceq, v)\right\}$. Hence, it remains to show that $\sigma(N, \preceq, v)$ contains an element $x$ such that $x_{k}=\alpha$. Let $x^{\prime}$ arise from $x$ by replacing $x_{k}$ by $y_{k}$ and $x_{\ell}$ by $x_{\ell}+x_{k}-y_{k}$. Then $x^{\prime} \in \sigma(N, \preceq, v)$ as we have shown. Now, applying RCP to $\{k, \ell\}$ allows us to reverse this modification so that $x \in \sigma(N, \preceq, v)$.

q.e.d.

\section{The prenucleolus and the bounded positive core}

This section is devoted to the characterization of the bounded positive core. Let $(N, \preceq, v) \in \Gamma, \mathcal{F}=$ $\mathcal{O}(N, \preceq)$, and $\mathcal{R}=\mathcal{R}^{(N, \preceq)}$. We may rewrite $(3.3)$ as

$$
C_{+}^{b}(N, \preceq, v)=\left\{x \in C_{+}(N, \preceq, v) \mid \max \{e(S, x, v) \mid S \in \mathcal{F}, \ell \notin S \ni k\}=0 \text { for all } k, \ell \in N, k \prec \cdot \ell\right\} .
$$

Note that $C_{+}^{b}(\cdot)$ inherits the following properties from $C_{+}(\cdot)$ : NE, PO, COV, AN, REAS, and CLOS. We now define a particular subsolution of $C_{+}^{b}(\cdot)$ that is single-valued and satisfies ND. Let $\mathcal{F}^{*}=\mathcal{O}\left(N, \preceq^{*}\right)$ where $\left(N, \preceq^{*}\right)$ is the reverse order of $(N, \preceq)$. Moreover, denote by $\nu$ the prenucleolus of the intermediate game $\left(\mathcal{R}, v_{\mathcal{R}}\right)$. The prenucleolus of $(N, \preceq, v)$ is the set

$$
\mathcal{N}(N, \preceq, v)=\mathcal{N} \mathcal{U C}\left(\left(e\left(S, \cdot, v^{*}\right)\right)_{S \in \mathcal{F}^{*}},\left\{y \in C_{+}(N, \preceq, v) \mid y(Q)=\nu_{Q} \text { for } Q \in \mathcal{R}\right\}\right) .
$$

Proposition 4.1 For any $(N, \preceq, v) \in \Gamma, \mathcal{N}(N, \preceq, v)$ is a singleton that is contained in $C_{+}^{b}(N, \preceq, v)$.

Proof: Let $y \in C_{+}(N, \preceq, v)$ such that $y(Q)=\nu_{Q}$ for all $Q \in \mathcal{R}$ and define $\mu^{*}=\max \left\{e\left(S, y, v^{*}\right) \mid S \in\right.$ $\left.\mathcal{F}^{*}\right\}, \mu=\max \{e(S, y, v) \mid S \in \mathcal{F}\}$, and

$$
X=\left\{x \in C_{+}(N, \preceq, v) \mid x(Q)=\nu_{Q} \text { for all } Q \in \mathcal{R} \text { and } e\left(S, x, v^{*}\right) \leqslant \mu^{*} \text { for all } S \in \mathcal{F}^{*}\right\} .
$$

Then $X \neq \emptyset$ because $y \in X$. Therefore

$$
\mathcal{N}(N, \preceq, v)=\mathcal{N} \mathcal{U C}\left(\left(e\left(S, \cdot, v^{*}\right)\right)_{S \in \mathcal{F}^{*}}, X\right)
$$

Moreover, $X$ is closed and convex. We now show that $X$ is bounded. For $i \in N$ let $h(i)$ the height of $i$ w.r.t. $(N, \preceq)$. Let $x \in X$. If $h(i)=0$, then $i \in \mathcal{F}$ and, hence, $x_{i} \geqslant v(\{i\})-\mu$. Moreover, $N \backslash\{i\} \in \mathcal{F}^{*}$ so that $x(N \backslash\{i\}) \geqslant v^{*}(N \backslash\{i\})-\mu^{*}$, i.e., $x_{i} \leqslant \mu^{*}+v(\{i\})$. Assume now that we have shown already that all $x_{i}$ with $h(i)<k$ for some $k \in \mathbb{N}$ are bounded. If, now, $h(i)=k$, then let $S=\{j \in N \mid j \preceq i\}$. On the one hand, $x_{i} \geqslant v(S)-\mu-x(S \backslash\{i\})$, hence $x_{i}$ has a lower bound because $h(j)<k$ for all $j \in S \backslash\{i\}$ so 
that the inductive hypothesis can be applied. On the other hand $x_{i} \leqslant \mu^{*}+v(S)-x(S \backslash\{i\})$ so that $x_{i}$ is bounded from above.

As the excess functions are continuous and convex, and as $X$ is nonempty, compact, and convex, the prenucleolus $\mathcal{N} \mathcal{U C}\left(\left(e\left(S, \cdot, v^{*}\right)\right)_{S \in \mathcal{F}^{*}}, X\right)$ is a nonempty convex set such that $e\left(S, x, v^{*}\right)=e\left(S, x^{\prime}, v^{*}\right)$ for all $S \in \mathcal{F}^{*}$ and (Justman 1977) all $x, x^{\prime} \in \mathcal{N}(N, \preceq, v)$. By induction on $h^{*}(i)$, the height of $i$ w.r.t. $\left(N, \preceq^{*}\right)$ we show that $x_{i}=x_{i}^{\prime}$. Indeed, if $h^{*}(i)=0$, then $\{i\} \in \mathcal{F}^{*}$ so that $e\left(\{i\}, x, v^{*}\right)=e\left(\{i\}, x^{\prime}, v^{*}\right)$ implies $x_{i}=x_{i}^{\prime}$. If $h^{*}(i)>0$, then, with $S=\left\{j \in N \mid j \preceq^{*} i\right\}$,

$$
e\left(S, x, v^{*}\right)=v^{*}(S)-x(S \backslash\{i\})-x_{i}=v^{*}(S)-x^{\prime}(S \backslash\{i\})-x_{i}^{\prime}=e\left(S, x^{\prime}, v^{*}\right)
$$

so that $x_{i}=x_{i}^{\prime}$ by the inductive hypothesis applied to $j \in S \backslash\{i\}$. Let $\nu(N, \preceq, v)=\widehat{\nu}$ denote the unique element of $\mathcal{N}(N, \preceq, v)$. Moreover, let $k, \ell \in N$ such that $k \prec \cdot \ell$. Assume that $t=\max \{e(S, \widehat{\nu}, v) \mid S \in$ $\mathcal{F}, \ell \notin S \ni k\}<0$. Let $0<\varepsilon<-t$ and let $x \in \mathbb{R}^{N}$ only differ from $\widehat{\nu}$ inasmuch as $x_{k}=\widehat{\nu}_{k}-\varepsilon$ and $x_{\ell}=\widehat{\nu}_{\ell}+\varepsilon$. Then $x \in X$ and $e\left(T, x, v^{*}\right)<e\left(T, \widehat{\nu}, v^{*}\right)$ for all $T \in \mathcal{F}^{*}$ such that $k \notin T \ni \ell$ and $e\left(T, x, v^{*}\right)=e\left(T, \widehat{\nu}, v^{*}\right)$ for all other $T \in \mathcal{F}^{*}$ which is a contradiction. By $(4.1), \widehat{\nu} \in C_{+}^{b}(N, \preceq, v)$. q.e.d.

Remark 4.2 The solution $\mathcal{N}(\cdot)$ satisfies ND because it is single-valued and satisfies AN.

We provide now a combinatorial characterization of the prenucleolus of $(N, \preceq, v)$ by a Kohlberg-like criterion. To this end, we introduce the following collections, for any $Q \in \mathcal{R}$ :

$$
\begin{aligned}
\mathcal{D}^{\prime}\left(\alpha, x, v^{*}, Q\right) & =\left\{S \cap Q \mid S \in \mathcal{F}_{0}^{*}, e\left(S, x, v^{*}\right) \geqslant \alpha\right\} \\
\mathcal{E}(x, v, Q) & =\left\{T \cap Q \mid T \in \mathcal{F}_{0}, x(T)=v(T)\right\} \cup\{Q\},
\end{aligned}
$$

where $\mathcal{F}_{0}^{*}=\mathcal{O}_{0}\left(N, \preceq^{*}\right)$. For two collections $\mathcal{B}, \mathcal{B}^{\prime}$ in $2^{N}$ such that $\mathcal{B} \subseteq \mathcal{B}^{\prime}$, we say that $\mathcal{B}$ is balanced within $\mathcal{B}^{\prime}$ if there exists a collection $\mathcal{B} \subseteq \mathcal{C} \subseteq \mathcal{B}^{\prime}$ which is balanced.

Proposition 4.3 Let $(N, \preceq, v) \in \Gamma$, and consider $x \in C_{+}(N, \preceq, v)$ s.t. $x(Q)=\nu_{Q}$ for $Q \in \mathcal{R}$, where $\nu$ is the prenucleolus of $\left(\mathcal{R}, v_{\mathcal{R}}\right)$. The following are equivalent:

(1) $x=\mathcal{N}(N, \preceq, v)$

(2) For $\alpha>0, Q \in \mathcal{R}, y \in \mathbb{R}^{Q}$, with $y(Q)=0$ and $y(S) \leqslant 0$ for all $S \in \mathcal{D}^{\prime}\left(\alpha, x, v^{*}, Q\right) \cup \mathcal{E}(x, v, Q)$, we have $y(S)=0$ for all $S \in \mathcal{D}^{\prime}\left(\alpha, x, v^{*}, Q\right)$.

(3) For $\alpha>0$ and $Q \in \mathcal{R}, \mathcal{D}^{\prime}=\mathcal{D}^{\prime}\left(\alpha, x, v^{*}, Q\right)$ is balanced within $\mathcal{D}^{\prime} \cup \mathcal{E}(x, v, Q)$.

Proposition 4.3 generalizes Kohlberg's (1971) characterization of the nucleolus. The equivalence between (1) and (2) is proved similarly as in the classical case. The equivalence between (2) and (3) which is an immediate consequence of Farkas' lemma occurs as a special case of Lemma 2.1 of Derks, Peters, and Sudhölter (2014) and is, e.g., explicitly proved by Derks and Peters (1998).

By Remark 4.2 and Proposition 4.1 the bounded positive core satisfies ND. Moreover, a careful inspection of the definition of the reduced game together with (4.1) show that, by Lemma 3.3, $C_{+}^{b}(\cdot)$ also inherits RGP from $C_{+}(\cdot)$. Thus, $C_{+}^{b}(\cdot)$ does not satisfy RCP if $|U|=\infty$ by Theorem 3.7. 
In order to characterize the bounded positive core, the following properties of a solution $\sigma$ on a set $\Gamma^{\prime} \subseteq \Gamma$ are useful. The solution $\sigma$ satisfies

- the converse reduced game property (CRGP) if for $(N, \preceq, v) \in \Gamma^{\prime}$ with $|N| \geqslant 2$ and $x \in X(N, v)$ the following condition holds: If, for every $S \subseteq N$ with $|S|=2,\left(S, \preceq, v_{S, x}\right) \in \Gamma^{\prime}$ and $x_{S} \in \sigma\left(S, \preceq, v_{S, x}\right)$, then $x \in \sigma(N, \preceq, v)$.

- the restricted converse reduced game property (RCRGP) if for $(N, \preceq, v) \in \Gamma^{\prime}$ and $x \in X(N, v)$, with $\mathcal{R}=\mathcal{R}^{(N, \preceq)}$ the following condition holds: If there exists $S \subseteq N$ such that $|S \cap Q|=1$ for all $Q \in \mathcal{R},\left(S, \preceq, v_{S, x}\right) \in \Gamma$, and $x_{S} \in \sigma\left(S, v_{S, x}\right)$, and if $(T \preceq, v) \in \Gamma^{\prime}$ and $x_{T} \in \sigma\left(T, \preceq, v_{T, x}\right)$, for any $T=\{k, \ell\} \subseteq N$ with $k \prec \cdot \ell$, then $x \in \sigma(N, \preceq, v)$;

- the unrestricted reconfirmation property $\left(\mathrm{RCP}^{\text {free }}\right)$ if the restriction of $\sigma$ to $\Gamma^{\prime} \cap \Gamma^{\text {free }}$ satisfies $\mathrm{RCP}$;

- boundedness (BOUND) if $\sigma(N, \preceq, v)$ is a bounded set for all $(N, \preceq, v) \in \Gamma^{\prime}$.

The property BOUND is self-explanatory. Note that for a solution on $\Gamma^{\text {free }}$, REAS implies BOUND, but in general such an implication does not exist.

RCRGP has not been used in the literature. It may be interpreted as follows. If every "block", i.e., every connected component, may select a representative so that the coalition of representatives $S$ is satisfied with the preimputation $x$ (i.e., $x_{S} \in \sigma\left(S, v_{S, x}\right)$ ) and if every pair of players consisting of a player $\ell$ and her immediate subordinate $k$ is also satisfied (i.e., $x_{T} \in \sigma\left(T, \preceq, v_{T, x}\right)$, where $T=\{k, \ell\}$ ), then no player in the grand coalition has an objection against $x$ (i.e., $x \in \sigma(N, \preceq, v)$ ).

As $C_{+}(\cdot)$ satisfied RCP,$C_{+}^{b}(\cdot)$ satisfies $\mathrm{RCP}$ on $\Gamma^{\text {free }}$, i.e., $C_{+}^{b}(\cdot)$ satisfies $\mathrm{RCP}$ free . By (3.3), it also satisfies BOUND.

The following result may be proved similarly to Lemma 3.6.

Lemma 4.4 The bounded positive core satisfies RCRGP.

Proof: Let $(N, \preceq, v) \in \Gamma, \mathcal{F}=\mathcal{O}(N, \preceq, v), \mathcal{R}=\mathcal{R}^{(N, \preceq)}$, and let $x \in X(N, v)$ such that $x_{\{k, \ell\}} \in$ $C_{+}^{b}\left(\{k, \ell\}, \preceq, v_{\{k, \ell\}, x}\right)$ for all $k, \ell \in N$ such that $k \prec \cdot \ell$ and such that there exists $S \subseteq N$ with $|Q \cap S|=1$ for all $Q \in \mathcal{R}$ and $x_{S} \in C_{+}^{b}\left(S, v_{S, x}\right)$. For any $i \in S$ denote $Q_{i}$ the connected component that contains $i$. Hence, the mapping $S \rightarrow \mathcal{R}, i \mapsto Q_{i}$ for all $i \in S$, is a bijection.

Let $k, \ell \in N$ such that $k \prec \cdot \ell$. Now, $v_{\{k, \ell\}, x}(\{k\})=v(T)-x(T \backslash\{k\})$ for some $T \in \mathcal{F}$ with $\ell \notin T \ni k$. As $x_{\{k, \ell\}} \in C_{+}^{b}\left(\{k, \ell\}, \preceq, v_{\{k, \ell\}, x}\right), 0=v(T)-x(T)=\max \{v(P)-x(P) \mid \ell \notin P \ni k, P \in \mathcal{F}\}$.

By (3.1) and (4.1) it suffices to show that $y=(x(Q))_{Q \in \mathcal{R}} \in C_{+}\left(\mathcal{R}, v_{\mathcal{R}}\right)$. Now, $x_{S} \in C_{+}\left(S, v_{S, x}\right)$. As in the proof of Lemma 3.6, $e\left(T, x_{S}, v_{S, x}\right)_{+}=e\left(\left\{Q_{i} \mid i \in T\right\}, y, v_{\mathcal{R}}\right)_{+}$for all $T \subseteq \mathcal{F}(S)$ so that the proof is finished by (3.5).

q.e.d.

We now formulate the main characterization result of this section. 
Theorem 4.5 The bounded positive core is the unique solution that satisfies NE, BOUND, COV, RGP, $\mathrm{RCP}^{\text {free }}$, ND, and RCRGP, provided $|U|=\infty$.

We postpone the proof and first show a useful variant of Lemma 3.5 and present useful remark.

Lemma 4.6 If $\sigma$ is a solution that satisfies BOUND, COV, and RGP, then, for any $k, \ell \in N$ such that $k \prec \cdot \ell$ with $P=\{k, \ell\}, \sigma\left(P, \preceq, v_{P, x}\right)=C^{b}\left(P, \preceq, v_{P, x}\right)$ for any $x \in \sigma(N, \preceq, v)$, which yields

$$
\max \{e(S, x, v) \mid \ell \notin S \ni k, S \in \mathcal{F}\}=0
$$

Proof: Let $u=v_{P, x}$. Define $y \in \mathbb{R}^{P}$ by $y_{k}=x_{k}-u(\{k\})$ and $y_{\ell}=x_{\ell}+u(\{k\})-u(P)$. By RGP and translation COV, $y \in \sigma(P, \preceq, 0)$. By scale COV, $t y \in \sigma(P, \preceq, 0)$ for $t>0$ so that, by BOUND, $y=0$, which yields $x_{k}=\max \{v(S \cup\{k\})-x(S) \mid k \notin S \in \mathcal{F}\}$.

q.e.d.

Remark 4.7 In the proof of Theorem 4.5 we shall employ the following stronger version of Theorem 4.1 of Orshan and Sudhölter (2010) in which REAS is replaced by BOUND: If $|U|=\infty$, then the unique solutions on $\Gamma^{\text {free }}$ that satisfy NE, BOUND, COV, RGP, and ND, are (a) the prenucleolus, (b) the positive core, and (c) the relative interior of the positive core. This result is Theorem 4.9 of Orshan and Sudhölter (2001). The article from 2010 is a modification of the discussion paper from 2001. Theorem 4.9 relies on the lengthy and technically sophisticated proof of Lemma 4.2, whereas when replacing BOUND by REAS, the proof of the corresponding lemma (Lemma 4.6) takes just a few lines so that the authors used the well-accepted REAS in their 2010 article basically to offer an easier reading. In the present context, when restrictions are possible, REAS does not imply BOUND.

Proof of Theorem 4.5: It remains to show the uniqueness part. Let $\sigma$ be a solution that satisfies the desired properties. By Lemmas 4.6 and 3.6, $\sigma$ is a subsolution of $C_{+}^{b}$. Thus, it remains to show that $C_{+}^{b}(N, \preceq, v) \subseteq \sigma(N, \preceq, v)$.

Claim: The solution $\sigma$ coincides with $C_{+}=C_{+}^{b}$ on $\Gamma^{\text {free }}$. Recall that $\left(M^{\prime}, \preceq^{\prime}, u^{\prime}\right) \in \Gamma$ of the proof of Theorem 3.7 is defined by $M^{\prime}=\{1,2,3\}, u^{\prime}(M)=2$ and $u^{\prime}(S)=0$ for $S \in \mathcal{F}^{\prime} \backslash\left\{M^{\prime}\right\}$, where $\mathcal{F}^{\prime}=\mathcal{O}\left(M^{\prime}, \preceq^{\prime}\right)$. Also, let $P=\{1,3\}, Q=\{2,3\}$, and $R=\{1,2\}$. By NE, there exists $x \in \sigma\left(M^{\prime}, \preceq^{\prime}, u^{\prime}\right)$. As $x \in C_{+}^{b}\left(M^{\prime}, \preceq^{\prime}, u^{\prime}\right), x_{1}=0$ (by $\left.(4.1)\right)$ and $0 \leqslant x_{3} \leqslant 2$. If $x_{3}>0$, then $\left(x_{1}, x_{3}\right) \in C_{+}\left(P, u_{P, x}^{\prime}\right) \backslash$ rint $C_{+}\left(P, u_{P, x}^{\prime}\right)$, and if $x_{3}=0$, then $\left(x_{2}, x_{3}\right) \in C_{+}\left(Q, u_{Q, x}^{\prime}\right) \backslash \operatorname{rint} C_{+}\left(Q, u_{Q, x}^{\prime}\right)$. Hence, our claim is valid by Remark 4.7 in any case.

Now we can finish the proof. Let $x \in C_{+}^{b}(N, \preceq, v)$ and choose any $S \subseteq N$ such that $|S \cap Q|=1$ for any $Q \in \mathcal{R}^{(N, \preceq)}$. Then $(S, \preceq)$ has height 0 so that the reduced game w.r.t. $S$ belongs to $\Gamma^{\text {free }}$. By RGP of $C_{+}^{b}, x_{S} \in C_{+}\left(S, v_{S, x}\right)$. Hence, by our claim $x_{S} \in \sigma\left(S, v_{S, x}\right)$. Moreover, if $k, \ell \in N$ satisfy $k \prec \cdot \ell$, then $\left(\{k, \ell\}, \preceq, v_{\{k, \ell\}, x}\right)$ has a single-valued bounded (positive) core by (4.1). By RGP of $C_{+}^{b}$, the unique element is $\left(x_{k}, x_{\ell}\right)$, i.e., $x_{k}=v_{\{k, \ell\}, x}(\{k\})$. Hence, $\left(x_{k}, x_{\ell}\right) \in \sigma\left(\{k, \ell\}, \preceq, v_{\{k, \ell\}, x}\right)$ by NE of $\sigma$. By RCRGP, $x \in \sigma(N, \preceq, v)$.

q.e.d. 
Remark 4.8 Assume that $|U|=\infty$. According to Sobolev (1975), the prenucleolus is the unique solution on $\Gamma^{\text {free }}$ that satisfies single-valuedness (SIVA), AN, COV, and RGP. For a solution that satisfies SIVA, however, RGP is equivalent to RCP. Moreover, Orshan (1993) showed that AN may be replaced by the equal treatment property (ETP), and Orshan and Sudhölter (2003) proved that the four axioms may be replaced by NE, ETP, COV, and RCP. However, a nonempty solution on $\Gamma$ that contains the prenucleolus for unrestricted games and satisfies RGP or RCP and COV, cannot coincide with the prenucleolus on $\Gamma^{\text {free }}$ as an easy analysis of the reduced games of $\left(M^{\prime}, \preceq^{\prime}, u^{\prime}\right)$ (the game used in the proofs of the characterizations) shows. Hence, in the foregoing sense there is no "prenucleolus" on $\Gamma$.

\section{Characterizing the positive prekernel and its bounded variant}

This section is devoted to generalize the definition and characterization (Sudhölter and Peleg 2000) of the positive prekernel to games with precedence constraints. Let $(N, \preceq, v) \in \Gamma, \mathcal{F}=\mathcal{O}(N, \preceq)$, and $\mathcal{R}=\mathcal{R}^{(N, \preceq)}$. For $x \in \mathbb{R}^{N}$ and $k, \ell \in N, k \neq \ell$, denote by $s_{k \ell}(x, v)$ the maximum surplus of $k$ over $\ell$ at $x$, defined by

$$
s_{k \ell}(x, v)=\sup \{e(S, x, v) \mid \ell \notin S \ni k\} .
$$

The positive prekernel of $(N, \preceq, v)$ is the set

$$
P K_{+}(N, \preceq, v)=\left\{x \in X(N, v) \mid s_{k \ell}(x, v) \leqslant s_{\ell k}(x, v)_{+} \text {for all } k, \ell \in N, k \neq \ell\right\} .
$$

If the height of $(N, \preceq)$ is not 0 , i.e., if restrictions are present, then the prekernel (an element $x$ of which, similarly as in (5.1), has to satisfy $\left.s_{k \ell}(x, v)=s_{\ell k}(x, v)\right)$ is empty. Indeed, if $k \prec \ell$, then $s_{k \ell}(x, v) \in \mathbb{R}$, but $s_{\ell k}(x, v)=\sup \emptyset=-\infty$. Hence, if $x \in P K_{+}(N, \preceq, v)$, then $s_{k \ell}(x, v) \leqslant 0$. We conclude that

$$
P K_{+}(N, \preceq, v)=\left\{x \in X(N, v) \mid(x(Q))_{Q \in \mathcal{R}} \in P K_{+}\left(\mathcal{R}, v_{\mathcal{R}}\right) \text { and } e(S, x, v) \leqslant 0 \text { for all } S \in \mathcal{F}_{0}\right\} .
$$

Thus, we define the bounded positive prekernel by

$$
P K_{+}^{b}(N, \preceq, v)=\left\{x \in P K_{+}(N, \preceq v) \mid \max \{e(S, x, v) \mid S \in \mathcal{F}, \ell \notin S \ni k\}=0 \text { for all } k, \ell \in N, k \prec \cdot \ell\right\} .
$$

Remark 5.1 On $\Gamma^{\text {free }}$ the positive prekernel is characterized by NE, AN, REAS, the weak reduced game property (WRGP) the definition of which differs from RGP only inasmuch as only reduced games w.r.t. coalitions $S \subseteq N$ with $|S| \leqslant 2$ are considered, CRGP, and weak unanimity for 2-person games (WUTPG) requiring that, applied to any 2-person game $(N, v)$, the solution contains $\{x \in X(N, v) \mid$ $x_{i} \geqslant v(\{i\})$ for $\left.i \in N\right\}$. (Sudhölter and Peleg 2000, Theorem 7.1)

We now show that the result mentioned in Remark 5.1 may be generalized to $\Gamma$. A solution $\sigma$ on $\Gamma^{\prime} \subseteq \Gamma$ satisfies WUTPG if for all $(N, \preceq, v) \in \Gamma^{\prime}$ with $|N|=2, \sigma(N, \preceq, v) \supseteq\left\{x \in X(N, v) \mid x_{i} \geqslant\right.$ $v(\{i\})$ for all minimal $i \in N\}$.

Proposition 5.2 The positive prekernel is characterized by NE, AN, REAS, WRGP, CRGP, and WUTPG. 
Proof: By Proposition 2.4 and non-emptiness of $P K_{+}(\cdot)$ on $\Gamma^{\text {free }}, P K_{+}(\cdot)$ satisfies NE, and clearly it satisfies AN and WUTPG. Moreover, REAS, RGP, and CRGP follow from (5.2) and the corresponding properties on $\Gamma^{\text {free }}$. In order to show the uniqueness part, let $\sigma$ be a solution that satisfies the desired properties. By WRGP applied to 1-person reduced games and REAS, $\sigma$ satisfies PO. Moreover, for any $(N, v) \in \Gamma^{\text {free }}, \sigma(N, v)=P K_{+}(N, v)$ by the mentioned classical result. If $(N, \preceq, v) \in \Gamma \backslash \Gamma^{\text {free }}$, then $|N| \geqslant 2$. If $|N|=2$, then by PO and REAS, $\sigma(N, \preceq, v) \subseteq P K_{+}(N, \preceq, v)$. By WUTPG, $\sigma(N, \preceq, v)=$ $P K_{+}(N, \preceq, v)$. If $|N|>2$ and $x \in \sigma(N, \preceq, v)$, then, by WRGP of $\sigma, x_{S} \in \sigma\left(S, \preceq, v_{S, x}\right)$, and hence, $x_{S} \in P K_{+}\left(S, \preceq, v_{S, x}\right)$, for all $S \subseteq N$ with $|S|=2$. By CRGP of $P K_{+}(\cdot), x \in P K_{+}(N, \preceq, v)$. The opposite inclusion follows similarly by interchanging the roles of $\sigma$ and $P K_{+}$.

q.e.d.

The bounded positive prekernel inherits NE, AN, REAS, RGP, and CRGP from the positive prekernel. Hence, the bounded variant exclusively violates WUTPG of the axioms in Proposition 5.2, i.e., $P K_{+}^{b}(N, \preceq, v)$ is a singleton whenever $|N|=2$ and $(N, \preceq)$ has not height 0 . Hence, we note that the bounded positive prekernel is characterized by NE, AN, REAS, WRGP, CRGP, WUTPG free (requiring that the core is contained in the solution applied to any classical 2-person game), and BOUND.

Here, REAS cannot be deleted. Indeed, let $t>0$ and, define the following solution $\sigma$ by the requirement that $x \in \sigma(N, \preceq, v)$ if $s_{k \ell}(x, v) \leqslant\left(s_{\ell k}(x, v)\right)_{+}$for all $k, \ell \in N, k \neq \ell$, and $\max _{i \in S \not j, S \in \mathcal{F}} e(S, x, v)=0$ for all $i, j \in N, i \prec j$, and $v(N)-t \leqslant x(N) \leqslant v(N)$, then $P K_{+}^{b}(\cdot)$ is a subsolution of $\sigma$ that, hence, satisfies NE and WUTPG ${ }^{\text {free }}$. Also, it satisfies BOUND and AN and the remaining properties (WRGP and CRGP) are easily deduced as well. However, $\sigma$ does not coincide with $P K_{+}^{b}(\cdot)$ already for 1-person games.

\section{On the logical independence of the employed axioms}

By generalizing or slightly modifying the solution concepts $\sigma^{1}, \ldots, \sigma^{5}$ of Orshan and Sudhölter (2010, Sect. 4.1), we will show that each of the axioms (1) NE, (2) REAS (respectively, BOUND), (3) COV, (4) RGP, (5) RCP (respectively, RCP free) in Theorem 3.7 or Theorem 4.5, respectively, is logically

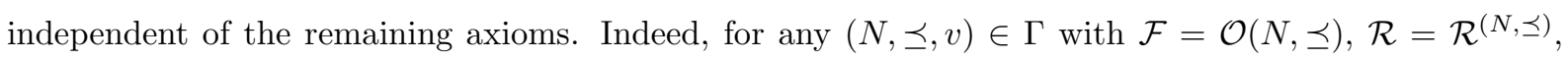
and $\mu=\max _{\mathcal{T} \subseteq \mathcal{R}} e\left(\mathcal{T}, \nu\left(\mathcal{R}, v_{\mathcal{R}}\right), v_{\mathcal{R}}\right)$, let

$$
\begin{aligned}
& \sigma^{1}(N, \preceq, v)=C(N, \preceq, v) \\
& \sigma^{1 b}(N, \preceq, v)=C^{b}(N, \preceq, v) \text {; } \\
& \sigma^{2}(N, \preceq, v)=X(N, \preceq, v) \text {; } \\
& \sigma^{3}(N, \preceq, v)=\mathcal{N} \mathcal{U C}\left((\max \{e(S, \cdot, v), t\})_{S \in \mathcal{F}}, X(N, v)\right) \text { for some } t<0 ; \\
& \sigma^{3 b}(N, \preceq, v)=\left\{x \in \sigma^{3}(N, \preceq, v) \mid \max \{e(S, x, v) \mid S \in \mathcal{F}, \ell \notin S \ni k\}=t \text { for all } k, \ell \in N, k \prec \cdot \ell\right\} \text {; } \\
& \sigma^{4}(N, \preceq, v)=\left\{x \in X(N, v) \mid e\left(\mathcal{T},(x(Q))_{Q \in \mathcal{R}}, v_{\mathcal{R}}\right) \leqslant \mu \text { for } \mathcal{T} \subseteq \mathcal{R} \text { and } e(S, x, v) \leqslant 0 \text { for } S \in \mathcal{F}_{0}\right\} ; \\
& s^{4 b}(N, \preceq, v)=\left\{x \in \sigma^{4}(N, \preceq, v) \mid \max \{e(S, x, v) \mid S \in \mathcal{F}, \ell \notin S \ni k\}=0 \text { for all } k, \ell \in N, k \prec \cdot \ell\right\} ; \\
& \sigma^{5}(N, \preceq, v)=P K_{+}(N, \preceq, v) \text {; and } \\
& \sigma^{5 b}(N, \preceq, v)=P K_{+}^{b}(N, \preceq, v) .
\end{aligned}
$$


Then it is easy to check that $\sigma^{i}$ satisfies all axioms from (1) to (5) except (i), for all $i=1, \ldots, 5$, and similarly for $\sigma^{i b}$ and the bounded version of the axioms. Also, as mentioned, rint $C_{+}$satisfies all axioms of Theorem 3.7 except CLOS. The solutions $\sigma^{1}$ and $\sigma^{1 b}$, respectively, also show that NE is logically independent in the characterization of the positive prekernel (Proposition 5.2) and its bounded variant. Similarly, the (bounded) positive core has all properties except CRGP and the correspondence $X$ only violates REAS in Proposition 5.2. The solution that assigns the positive prekernel to each $(N, \preceq, v)$ with $|N| \leqslant 2$ and otherwise the set of all reasonable preimputations only violates WRGP, and its bounded variant may be used to show that RGP is independent of the remaining axioms in the characterization of the bounded positive prekernel. We now generalize the definition of the solution of Example 8.3 of the aforementioned article. Choose two players, say 1 and 2 and define, for all $(N, \preceq, v) \in \Gamma, \sigma^{6}(N, \preceq, v)=$ $P K_{+}(N, \preceq, v)$ if $|N| \leq 2$ and $\{1,2\} \nsubseteq N$ or $C(N, \preceq, v) \neq \emptyset$. If $N=\{1,2\}$ and $C(N, \preceq, v)=\emptyset$ (hence the height of $(N, \preceq)$ is 0$)$, put $\sigma^{6}(N, \preceq, v)=P K(N, v) \cup\{(v(\{1\}), v(N)-v(\{1\})),(v(N)-v(\{2\}), v(\{2\}))\}$. For $|N| \geqslant 3$, put $\sigma^{6}(N, \preceq, v)=\left\{x \in X(N, v) \mid x_{S} \in \sigma^{6}\left(S, \preceq, v_{S, x}\right)\right.$ for all $\left.S \subseteq N,|S|=2\right\}$ and let $\sigma^{6 b}$ be its bounded pendant. Hence,

$$
\begin{aligned}
\sigma^{6}(N, \preceq, v) & =\left\{x \in X(N, v) \mid(x(Q))_{Q \in \mathcal{R}} \in \sigma^{6}\left(\mathcal{R}, v_{\mathcal{R}}\right), e(S, x, v) \leqslant 0 \text { for all } S \in \mathcal{F}_{0}\right\} \text { and } \\
\sigma^{6 b}(N, \preceq, v) & =\left\{x \in \sigma^{6}(N, \preceq, v) \mid \max \{e(S, x, v) \mid S \in \mathcal{F}, \ell \notin S \ni k\}=0 \text { for all } k, \ell \in N, k \prec \cdot \ell\right\} .
\end{aligned}
$$

As in the classical case, $\sigma^{6}$ (respectively, $\sigma^{6 b}$ ) satisfies NE, REAS (respectively, BOUND), RGP, CRGP, and WUTPG (respectively, WUTPG ${ }^{\text {free }}$ ); and it violates AN.

Let $t>0$ and

$$
\sigma^{7}(N, \preceq, v)=\left\{x \in X(N, v) \mid s_{k \ell}(x, v) \leqslant \max \left\{-t, s_{\ell k}(x, v)\right\} \text { for all } k, \ell \in N, k \neq \ell\right\} .
$$

Then $\sigma^{7}$ exclusively violates WUTPG and the variant defined by

$$
\sigma^{7 b}(N, \preceq, v)=\left\{x \in \sigma^{6}(N, \preceq, v) \mid \max \{e(S, x, v) \mid S \in \mathcal{F}, \ell \notin S \ni k\}=-t \text { for all } k, \ell \in N, k \prec \cdot \ell\right\}
$$

satisfies the remaining axioms of the bounded positive prekernel.

We don't know if ND is logically independent of the remaining axioms in the characterizing result of the (bounded) positive core. We remark that ND is logically independent of NE, REAS (BOUND), COV, and RGP on $\Gamma^{\text {free }}$ (Orshan and Sudhölter 2010), i.e., there are solutions that satisfy NE, REAS, COV, and RGP, and do neither coincide with the prenucleolus nor with the positive core or its relative interior. But as soon as an axiom is added that guarantees that the positive core is the only solution that has also this property, then it is an open problem whether or not ND is still needed.

Moreover, we have to admit that we don't know whether RCRGP is logically independent of the remaining axioms in Theorem 4.5.

Finally, examples of the mentioned paper may be generalized to show that the infinity assumption on $|U|$ is necessary in the characterization of the (bounded) positive core and that for $|U|=2$ the statement of Proposition 5.2 is no longer valid. 


\section{References}

Algaba, E., J. M. Bilbao, P. Borm, and J. J. López (2000): "The position value for union stable systems", Mathematical Methods of Operations Research, 52, 221 - 236.

Algaba, E., J. M. Bilbao, R. van den Brink, and A. Jiménez-Losada (2004): "Cooperative games on antimatroids", Discrete Mathematics, 282, 1 - 15 .

Aumann, R. J., AND J. H. DrÈze (1974): "Cooperative games with coalition structures", International Journal of Game Theory, 3, 217 - 237.

Aumann, R. J., And M. Maschler (1964): "The bargaining set for cooperative games", in Advances in Game Theory, ed. by M. Dresher, L. S. Shapley, and A. W. Tucker, Vol. 52 of Annals of Mathematical Studies, pp. 443 - 476, Princeton, N.J. Princeton University Press.

Bilbao, J. M., E. Lebrón, And N. JimÉnez (1998): "Probabilistic values on convex geometries", Annals of Operations Research, 84, $79-95$.

DAVIS, M., AND M. MAschler (1967): "Existence of stable payoff configurations for cooperative games", in Essays in Mathematical Economics in Honor of Oskar Morgenstern, ed. by M. Shubik, pp. 39 - 52, Princeton, N.J. Princeton University Press.

Derks, J. J. M., ANd R. P. Gilles (1995): "Hierarchical organization structures and constraints on coalition formation", International Journal of Game Theory, 24, 147 - 163.

Derks, J. J. M., And H. Peters (1998): "Orderings, excess functions, and the nucleolus", Mathematical Social Sciences, 36, $175-182$.

Derks, J. J. M., H. Peters, and P. Sudhölter (2014): "On extensions of the core and the anticore of transferable utility games", International Journal of Game Theory, 43, 37 - 63.

FaIGLE, U. (1989): "Cores of games with restricted cooperation", Zeitschrift für Operations ResearchMathematical Methods of Operations Research, 33, $405-422$.

Faigle, U., And W. Kern (1992): "The Shapley value for cooperative games under precedence constraints", International Journal of Game Theory, 21, 249 - 266.

Fujishige, S. (2005): Submodular functions and optimization, Vol. 58 of Annals of Discrete Mathematics. Elsevier, Amsterdam, 2nd edn.

Gilles, R. P., G. Owen, and R. van den Brink (1992): "Games with permission structures: the conjunctive approach", International Journal of Game Theory, 20, 277 - 293.

Grabisch, M. (2011): "Ensuring the boundedness of the core of games with restricted cooperation", Annals of Operations Research, 191, 137 - 154.

(2013): "The core of games on ordered structures and graphs", Annals of Operations Research, $204,33-64$.

Grabisch, M., And P. Sudhölter (2012): "The bounded core for games with precedence constraints", Annals of Operations Research, 201, $251-264$.

(2014): "On the restricted cores and the bounded core of games on distributive lattices", European Journal of Operational Research, 235, $709-717$.

Grabisch, M., and L. J. XIE (2007): "A new approach to the core and Weber set of multichoice games", Mathematical Methods of Operations Research, 66, $491-512$.

Granot, D. (1994): "On a new bargaining set for cooperative games", Working paper, Faculty of Commerce and Business Administration, University of British Columbia. 
ICHIISHI, T. (1981): "Super-modularity: Applications to convex games and to the greedy algorithm for LP", Journal of Economic Theory, 25, 283 - 286.

Justman, M. (1977): "Iterative processes with 'nucleolar' restrictions", International Journal of Game Theory, 6, $189-212$.

KoHlBerg, E. (1971): "On the nucleolus of a characteristic function game", SIAM Journal on Applied Mathematics, 20, $62-66$.

Lange, F., And M. Grabisch (2009): "Values on regular games under Kirchhoff's laws", Mathematical Social Sciences, 58, $322-340$.

Mas-Colell, A. (1989): "An equivalence theorem for a bargaining set", Journal of Mathematical Economics, 18, $129-139$.

Orshan, G. (1993): "The prenucleolus and the reduced game property: Equal treatment replaces anonymity", International Journal of Game Theory, 22, 241 - 248.

Orshan, G., And P. Sudhölter (2001): "The positive core of a cooperative game", Discussion paper 268, Center for Rationality and Interactive Decision Theory, The Hebrew University of Jerusalem.

- (2003): "Reconfirming the prenucleolus", Mathematics of Operations Research, 28, 283 - 293.

$113-136$

Owen, G. (1977): "Values of games with a priori unions", in Essays in Mathematical Economics and Game Theory, ed. by R. Henn, and O. Moeschlin, pp. 76 - 88, New York. Springer-Verlag.

PeleG, B. (1986): "On the reduced game property and its converse", International Journal of Game Theory, 15, $187-200$.

Peleg, B., And P. Sudhölter (2007): Introduction to the Theory of Cooperative Games, Theory and Decisions Library, Series C: Game Theory, Mathematical Programming and Operations Research. Springer-Verlag, Berlin, Heidelberg, 2nd edn.

Rockafellar, R. T. (1970): Convex Analysis. Princeton University Press, New Jersey.

SChmeIDLER, D. (1969): "The nucleolus of a characteristic function game", SIAM Journal on Applied Mathematics, 17, 1163 - 1170.

Shapley, L. S. (1971): "Cores of convex games", International Journal of Game Theory, 1, 11 - 26.

Sobolev, A. I. (1975): "The characterization of optimality principles in cooperative games by functional equations", in Mathematical Methods in the Social Sciences, ed. by N. N. Vorobiev, Vol. 6, pp. 95 151, Vilnius. Academy of Sciences of the Lithuanian SSR, in Russian.

Sudhölter, P., and B. Peleg (2000): "The positive prekernel of a cooperative game", International Game Theory Review, 2, $287-305$.

Sudhölter, P., And J. A. M. Potters (2001): "The semireactive bargaining set of a cooperative game", International Journal of Game Theory, 30, 117 - 139.

Tomizawa, N. (1983): "Theory of hyperspace (XVI) — on the structure of hedrons", Papers of the Technical Group on Circuits and Systems CAS82-172, Inst. of Electronics and Communications Engineers of Japan, In Japanese.

VAN DEN BRINK, R., AND R. Gilles (1996): "Axiomatizations of the conjunctive permission value for games with permission structures", Games and Economic Behavior, 12, 113 - 126. 